\title{
DYNAMIC ALPHA-INVARIANTS OF DEL PEZZO SURFACES
}

\author{
IVAN CHELTSOV AND JESUS MARTINEZ-GARCIA
}

\begin{abstract}
For every smooth del Pezzo surface $S$, smooth curve $C \in$ $\left|-K_{S}\right|$ and $\beta \in(0,1]$, we compute the $\alpha$-invariant of Tian $\alpha(S,(1-$ $\beta) C$ ) and prove the existence of Kähler-Einstein metrics on $S$ with edge singularities along $C$ of angle $2 \pi \beta$ for $\beta$ in certain interval. In particular we give lower bounds for the invariant $R(S, C)$, introduced by Donaldson as the supremum of all $\beta \in(0,1]$ for which such a metric exists. The pairs $(S, C)$ considered are strongly asymptotically log del Pezzo surfaces. We study one of the two classes of such pairs for which such metrics are expected to exist for all small $\beta>0$.
\end{abstract}

\section{INTRODUCTION}

In the last fifty years, the existence of canonical metrics on complex manifolds has attracted a lot of interest from complex differential geometers. In the case of Fano manifolds the Yau-Tian-Donaldson conjecture (recently solved by Chen, Donaldson, Sun [6] and Tian [21]) predicted that the existence of Kähler-Einstein metrics should be equivalent to the algebrogeometric concept of $K$-stability.

In recent decades, many geometric problems have been generalized to a log setting, initially in an attempt to solve these problems and later on their own merit. This was indeed the approach successfully suggested by Donaldson to solve the Kähler-Einstein problem (see [9]). In this article, we study the Kähler-Einstein problem for surfaces in a natural log setting:

Definition $1.1([5])$. Let $\left(X, D=\sum_{i=1}^{r} D_{i}\right)$ be $\log$ smooth, i.e. $X$ is non-singular and $D$ is a reduced $\mathbb{Z}$-divisor whose irreducible components are smooth and intersect with simple normal crossings. Consider $\beta=$ $\left(\beta_{1}, \ldots, \beta_{r}\right) \in(0,1]^{r}$. The pair $(X, D)$ is strongly asymptotically log Fano if for all sufficiently small $0<\|\beta\| \ll 1$, the $\mathbb{R}$-divisor $-\left(K_{X}+\sum\left(1-\beta_{i}\right) D_{i}\right)$ is ample.

Strongly asymptotically log Fano varieties are a natural generalization of $\log$ Fano varieties $(X, D)$, for which $-\left(K_{X}+D\right)$ is ample. Furthermore, both concepts generalize Fano varieties. Indeed, a (strongly asymptotically) $\log$ Fano variety $(X, D)$ such that $D=0$ is a Fano variety.

Given a strongly asymptotically log Fano pair $(X, D)$, we may consider Kähler-Einstein metrics on $X$ with edge singularities (KEE metrics) of angles $2 \pi \beta_{1}, \ldots, 2 \pi \beta_{r}$ along $D_{1}, \ldots, D_{r}$, respectively. In this log setting, the 
Kähler-Einstein edge problem consists of two parts. On the one hand we must decide if $(X, D)$ admits a KEE metric for all small values of $\|\beta\|$. On the other hand, it is interesting to describe the geometry of the space of $\beta \in(0,1]^{r}$ such that $\left(X, \sum\left(1-\beta_{i}\right) D_{i}\right)$ admits a KEE metric, and in particular on the boundary of this space.

The particular case when $r=1$ (i.e. $\operatorname{Supp}(D)$ consists of one smooth component) and $D \in\left|-K_{X}\right|$ is of special interest, since a Kähler-Einstein metric with singularities along $D$ of angle $2 \pi$ is a Kähler-Einstein metric in the usual sense. Moreover, in this case we can pick any $\beta \in(0,1]$, since $-\left(K_{X}+(1-\beta) D\right) \sim_{\mathbb{Q}}-\beta K_{X}$ is ample. Hence, in that case, studying the geometry of $\beta \in(0,1]$ is equivalent to computing the following invariant introduced by Donaldson:

Definition $1.2([9])$. Let $X$ be a smooth Fano variety, and let $D$ be a smooth divisor in $\left|-K_{X}\right|$. Then $R(X, D)$ is the supremum of all $\beta \in(0,1]$ such that $X$ admits a Kähler-Einstein metric with edge singularities along $D$ of angle $2 \pi \beta$.

It follows from [10] that the smooth Fano variety $X$ admits a KählerEinstein metric with edge singularities of angle $2 \pi \beta$ along $D$ for every positive $\beta<R(X, D)$.

Remark 1.3. If $X$ is a smooth Fano variety, $D \in\left|-K_{X}\right|$ is a smooth divisor and $X$ admits a Kähler-Einstein metric, then $R(X, D)=1$.

Cheltsov and Rubinstein [5] have classified all strongly asymptotically $\log$ Fano varieties $(S, D)$ in dimension 2. In their classification, $(S, D)$ is conjectured to admit KEE metrics for all small $0<\beta \ll 1$ [5, Conjecture 1.6.] if and only if $\left(K_{S}+D\right)^{2}=0$. The main subcase of this classification, and the focus of this article, is the case in which $S$ is a del Pezzo surface ( $-K_{S}$ is ample) and $D \in\left|-K_{S}\right|$ is a smooth curve. This is the natural setting in which $R(S, D)$ above is defined. Before explaining our contribution, let us discuss the main tool used: Tian's $\alpha$-invariant.

Let $(V, \Delta)$ be a $\log$ Fano variety, where $\Delta$ is an $\mathbb{R}$-divisor. Its $\alpha$-invariant can be defined as

$\alpha(V, \Delta)=\sup \left\{\begin{array}{l|l}\lambda \in \mathbb{R} & \begin{array}{l}\text { the log pair }(V, \Delta+\lambda B) \text { is log canonical } \\ \text { for any effective } \mathbb{R} \text {-divisor } B \sim_{\mathbb{R}}-\left(K_{V}+\Delta\right)\end{array}\end{array}\right\} \in \mathbb{R}_{>0}$.

If $\Delta=0$, we denote $\alpha(V, \Delta)$ by $\alpha(V)$.

Remark 1.4. For every effective $\mathbb{R}$-Cartier $\mathbb{R}$-divisor $B$ on $V$, the number

$$
\operatorname{lct}(V, \Delta ; B)=\sup \{\lambda \in \mathbb{R} \mid \text { the } \log \text { pair }(V, \Delta+\lambda B) \text { is } \log \text { canonical }\}
$$

is called the log canonical threshold of $B$ with respect to $(V, \Delta)$. Note that

$\alpha(V, \Delta)=\inf \left\{\operatorname{lct}(V, \Delta ; B) \mid B\right.$ is an effective $\mathbb{R}$-divisor such that $\left.B \sim_{\mathbb{R}}-\left(K_{V}+\Delta\right)\right\}$. 
The role of $\alpha$-invariants in the study of KEE metrics arises from the following theorem:

Theorem 1.5 ([10, Theorem 2, Lemma 6.13], c.f. [15, Theorem 5.4]). Let $X$ be a smooth projective variety of dimension $n$, and let $D$ be a smooth irreducible hypersurface in $X$. Let $\beta \in(0,1]$ and suppose that the divisor $-\left(K_{X}+(1-\beta) D\right)$ is ample. If $\alpha(X,(1-\beta) D)>\frac{n}{n+1}$, then $X$ admits a Kähler-Einstein metric with edge singularities of angle $2 \pi \beta$ along $D$.

The $\alpha$-invariants of smooth del Pezzo surfaces were computed in [1, Theorem 1.7] (see [7], [16] for an analytic approach and [14] for a characteristic free approach). The computation implies

Theorem 1.6. Let $S$ be a smooth del Pezzo surface. Then

$\alpha(S)=\inf \left\{\operatorname{lct}(S, 0 ; B)|B \in|-K_{S} \mid\right.$ and $B=\sum B_{i}$, where $B_{i} \cong \mathbb{P}^{1}$ and $\left.-K_{S} \cdot B_{i} \leqslant 3 \forall i\right\}$.

Let $X$ be a smooth Fano variety and $D \in\left|-K_{X}\right|$ be a smooth divisor. By the definition of $\alpha$-invariant, and Theorem 1.5 it follows that there is a KEE metric for all sufficiently small $0<\beta \ll 1$. On the other hand, it well known that the existence of KEE metrics is a convex property for $\beta$. Therefore $(X, D)$ admits a KEE metric for all $0<\beta \leqslant R(X, D)$.

By a theorem of Tian (see [20]), a smooth del Pezzo surface $S$ admits a Kähler-Einstein metric if and only if $S \neq \mathbb{F}_{1}$ and $K_{S}^{2} \neq 7$. Thus, we have

Corollary 1.7 ([20]). Let $S$ be a smooth del Pezzo surface such that $S ¥ \mathbb{F}_{1}$ and $K_{S}^{2} \neq 7$, and let $C$ be a smooth curve in $\left|-K_{S}\right|$. Then $R(S, C)=1$.

Unless $R(X, D)=1$, we do not know a single example for which the invariant $R(X, D)$ is known precisely (cf. [12, Theorem 1.7]).

In this article we generalize Theorem 1.6 to the case of strongly asymptotically $\log$ Fano surfaces $(S, C)$, where $S$ is a del Pezzo surface and $C \in\left|-K_{S}\right|$ is smooth.

Theorem 1.8 (Main Theorem). Let $S$ be a smooth del Pezzo surface, let $C$ be a smooth curve in $\left|-K_{S}\right|$, and let $\beta$ be a real number in $(0,1]$. Then

$\alpha(S,(1-\beta) C)=\inf \left\{\begin{array}{l|l}\operatorname{lct}(S,(1-\beta) C ; \beta B) & \begin{array}{l}B \in\left|-K_{S}\right| \text { such that } B=C \text { or } B=\sum B_{i}, \\ \text { where } B_{i} \cong \mathbb{P}^{1} \text { and }-K_{S} \cdot B_{i} \leqslant 3 \forall i\end{array}\end{array}\right\}$.

Moreover, we establish how the $\alpha$-invariants of del Pezzo surfaces vary under blow-ups:

Theorem 1.9. Let $S_{1}$ and $S_{2}$ be smooth del Pezzo surfaces, let $C_{1}$ and $C_{2}$ be smooth curves in $\left|-K_{S_{1}}\right|$ and $\left|-K_{S_{2}}\right|$, respectively. Suppose that there is a birational morphism $f: S_{2} \rightarrow S_{1}$ such that $f\left(C_{2}\right)=C_{1}$. Then $\alpha\left(S_{1},(1-\beta) C_{1}\right) \leqslant \alpha\left(S_{2},(1-\beta) C_{2}\right)$ for every $\beta \in(0,1]$ except for the following cases: 
(1) $S_{1} \cong \mathbb{P}^{2}, S_{2} \cong \mathbb{F}_{1}$, and $f$ is the blow up of an inflection point of the cubic curve $C_{1} \subset \mathbb{P}^{2}$,

(2) $S_{1} \cong \mathbb{P}^{1} \times \mathbb{P}^{1}, K_{S_{2}}^{2}=7$, and $f$ is the blow up of a point in $C_{1}$.

We will prove Theorems 1.8 and 1.9 in Section 4. In Section 2, we will give very explicit formulas for the invariant $\alpha(S,(1-\beta) C)$. Instead of presenting them here, let us consider their applications.

Corollary 1.10. Let $S$ be a smooth del Pezzo surface, and let $C$ be a smooth curve in $\left|-K_{S}\right|$. Then $\alpha(S,(1-\beta) C)$ is a decreasing continuous piecewise smooth function for $\beta \in(0,1]$.

If $S$ is a smooth del Pezzo surface such that either $S \cong \mathbb{F}_{1}$ or $K_{S}^{2}=7$, and $C$ is a smooth curve in $\left|-K_{S}\right|$, then $R(S, C) \geqslant \frac{1}{6}$ by [5, Proposition 6.10 (i)]. We improve this bound:

Corollary 1.11. Suppose that $S \cong \mathbb{F}_{1}$. Let $C$ be a smooth curve in $\left|-K_{S}\right|$. Then $R(S, C) \geqslant \frac{3}{10}$. Furthermore, if $C$ is chosen to be general in $\left|-K_{S}\right|$, then $R(S, C) \geqslant \frac{3}{7}$.

Corollary 1.12. Let $S$ be a smooth del Pezzo surface such that $K_{S}^{2}=7$, and let $C$ be a smooth curve in $\left|-K_{S}\right|$. Then $R(S, C) \geqslant \frac{3}{7}$. Furthermore, if $C$ does not pass through the intersection point of two intersecting $(-1)$-curves in $S$, then $R(S, C) \geqslant \frac{1}{2}$.

In $\left[22\right.$, Theorem 1], Székelyhidi proved that $R(S, C) \leqslant \frac{4}{5}$ when $S=\mathbb{F}_{1}$, and $R(S, C) \leqslant \frac{7}{9}$ when $K_{S}^{2}=7$ and $C$ passes through the intersection point of two intersecting $(-1)$-curves in $S$.

Structure of the article. In Section 2 we define explicit functions $\hat{\alpha}(S,(1-$ $\beta) C):(0,1] \rightarrow \mathbb{R}$ for all smooth del Pezzo surfaces $S$ and all smooth curves $C \in\left|-K_{S}\right|$. These functions coincide with specific values of $\operatorname{lct}(S,(1-$ $\beta) C, \beta B$ ) where $B \in\left|-K_{S}\right|$ and $B=C$ or $B=\sum B_{i}$ where $B_{i} \cong \mathbb{P}^{1}$ and $-K_{S} \cdot B_{i} \leqslant 3$ for all $i$.

The goal of this article is to prove Theorems 1.8 and 1.9. Both results follow from showing that $\alpha(S,(1-\beta) C)=\hat{\alpha}(S,(1-\beta) C)$ (see Theorems 4.1 and 4.10). Proving that this equality holds boils down to show that given any effective $\mathbb{R}$-divisor $D \sim_{\mathbb{R}}-K_{S}$ and any point $p \in S$, the pair

$$
(S,(1-\beta) C+\hat{\alpha}(S,(1-\beta) C) \beta D)
$$

is $\log$ canonical at $p$ for all $\beta \in(0,1]$.

The proof consists of several cases according to the pair $(S, C)$ and the position of $p \in S$. These are covered in Section 4. A particularly involved case is when $4 \leqslant K_{S}^{2} \leqslant 7, p$ belongs to a unique $(-1)$-curve $\mathcal{L}$ and $p=\mathcal{L} \cap C$. This case is treated separately in Section 5 . In Section 3 we provide a few local inequalities for pairs $(S, D)$ which are not log canonical. We use these inequalities in the proofs in sections 4 and 5 .

Most of the results in this article were obtained in the Ph.D. Thesis of the second author (see [13]). This article contains simplified proofs and, in 
some cases, completely new proofs. A part of this work was done during a visit of both authors to the National Center for Theoretical Sciences in Taipei. We are grateful to Jungkai Chen for making this visit possible.

Throughout this article, we assume that all considered varieties are projective and defined over $\mathbb{C}$.

\section{EXPLiCIT Formulas}

Let $S$ be a smooth del Pezzo surface. If $K_{S}^{2} \geqslant 3$, then $-K_{S}$ is very ample (see [11, Proposition III.3.4]. In this case, we will identify $S$ with its anticanonical image, and we will call a curve $Z \subset S$ such that $Z \cdot\left(-K_{S}\right)=$ $1,2,3$ a line, conic, cubic, respectively. Let $C$ be a smooth curve in $\left|-K_{S}\right|$, and let $\beta$ be a positive real number in $(0,1]$. Let

$\check{\alpha}(S,(1-\beta) C)=\inf \left\{\begin{array}{l|l}\operatorname{lct}(S,(1-\beta) C ; \beta B) & \begin{array}{l}B \in\left|-K_{S}\right| \text { such that } B=C \text { or } B=\sum B_{i}, \\ \text { where } B_{i} \cong \mathbb{P}^{1} \text { and }-K_{S} \cdot B_{i} \leqslant 3 \forall i\end{array}\end{array}\right\}$.

Then $\alpha(S,(1-\beta) C) \leqslant \check{\alpha}(S,(1-\beta) C)$. Theorem 1.8 states that $\alpha(S,(1-$ $\beta) C)=\check{\alpha}(S,(1-\beta) C)$. In this section, we will define a number $\hat{\alpha}(S,(1-\beta) C)$ such that $\hat{\alpha}(S,(1-\beta) C) \geqslant \check{\alpha}(S,(1-\beta) C)$. In Section 4 , we will prove that $\alpha(S,(1-\beta) C) \geqslant \hat{\alpha}(S,(1-\beta) C)$. The latter inequality implies Theorem 1.8, since $\hat{\alpha}(S,(1-\beta) C) \geqslant \check{\alpha}(S,(1-\beta) C) \geqslant \alpha(S,(1-\beta) C)$.

2.1. Projective plane. Suppose that $S \cong \mathbb{P}^{2}$. Then $C$ is a smooth cubic curve on $S$. Let

$$
\hat{\alpha}(S,(1-\beta) C)=\min \left\{1, \frac{1+3 \beta}{9 \beta}, \frac{1}{3 \beta}\right\}=\left\{\begin{array}{l}
1 \text { for } 0<\beta \leqslant \frac{1}{6}, \\
\frac{1+3 \beta}{9 \beta} \text { for } \frac{1}{6} \leqslant \beta \leqslant \frac{2}{3}, \\
\frac{1}{3 \beta} \text { for } \frac{2}{3} \leqslant \beta \leqslant 1 .
\end{array}\right.
$$

Let $P$ be an inflection point of the curve $C$, and let $T$ be the line in $\mathbb{P}^{2}$ that is tangent to $C$ at the point $P$. Then $\hat{\alpha}(S,(1-\beta) C) \geqslant \check{\alpha}(S,(1-\beta) C)$, since

$$
\hat{\alpha}(S,(1-\beta) C)=\min \{\operatorname{lct}(S,(1-\beta) C ; \beta C), \operatorname{lct}(S,(1-\beta) C ; 3 \beta T)\} .
$$

2.2. Smooth quadric surface. Suppose that $S \cong \mathbb{P}^{1} \times \mathbb{P}^{1}$. Let

$$
\hat{\alpha}(S,(1-\beta) C)=\min \left\{1, \frac{1+2 \beta}{6 \beta}\right\}=\left\{\begin{array}{l}
1 \text { for } 0<\beta \leqslant \frac{1}{4} \\
\frac{1+2 \beta}{6 \beta} \text { for } \frac{1}{4} \leqslant \beta \leqslant 1 .
\end{array}\right.
$$

Let $T$ be a divisor of bi-degree $(1,1)$ on $S$ that is a union of two fibers of each projection from $S$ to $\mathbb{P}^{1}$. Suppose in addition that one component of $T$ 
is tangent to $C$ at some point, and another component of $T$ passes through this point. Then $\hat{\alpha}(S,(1-\beta) C) \geqslant \check{\alpha}(S,(1-\beta) C)$, since

$$
\hat{\alpha}(S,(1-\beta) C)=\min \{\operatorname{lct}(S,(1-\beta) C ; \beta C), \operatorname{lct}(S,(1-\beta) C ; 2 \beta T)\} .
$$

2.3. First Hirzebruch surface. Suppose that $S \cong \mathbb{F}_{1}$. Let $Z$ be the unique $(-1)$-curve in $S$, and let $F$ be the fiber of the natural projection $S \rightarrow \mathbb{P}^{1}$ that passes through the point $C \cap Z$. Then $C \sim 2 Z+3 F$. If $F$ is tangent to $C$ at the point $C \cap Z$, let

$$
\hat{\alpha}(S,(1-\beta) C)=\min \left\{1, \frac{1+2 \beta}{8 \beta}, \frac{1}{3 \beta}\right\}=\left\{\begin{array}{l}
1 \text { for } 0<\beta \leqslant \frac{1}{6}, \\
\frac{1+2 \beta}{8 \beta} \text { for } \frac{1}{6} \leqslant \beta \leqslant \frac{5}{6}, \\
\frac{1}{3 \beta} \text { for } \frac{5}{6} \leqslant \beta \leqslant 1 .
\end{array}\right.
$$

If $F$ is not tangent to $C$ at the point $C \cap Z$, let

$$
\hat{\alpha}(S,(1-\beta) C)=\min \left\{1, \frac{1+\beta}{5 \beta}, \frac{1}{3 \beta}\right\}=\left\{\begin{array}{l}
1 \text { for } 0<\beta \leqslant \frac{1}{4}, \\
\frac{1+\beta}{5 \beta} \text { for } \frac{1}{4} \leqslant \beta \leqslant \frac{2}{3}, \\
\frac{1}{3 \beta} \text { for } \frac{2}{3} \leqslant \beta \leqslant 1 .
\end{array}\right.
$$

In both cases, we have $\hat{\alpha}(S,(1-\beta) C) \geqslant \check{\alpha}(S,(1-\beta) C)$, because

$$
\hat{\alpha}(S,(1-\beta) C)=\min \{\operatorname{lct}(S,(1-\beta) C ; \beta C), \operatorname{lct}(S,(1-\beta) C ; \beta(2 Z+3 F))\} \text {. }
$$

2.4. Blow up of $\mathbb{P}^{2}$ at two points. Suppose that $K_{S}^{2}=7$. Then there exists a birational morphism $\pi: S \rightarrow \mathbb{P}^{2}$ that is the blow up of two distinct points in $\mathbb{P}^{2}$. Denote by $E_{1}$ and $E_{2}$ two $\pi$-exceptional curves, and denote by $L$ the proper transform of the line in $\mathbb{P}^{2}$ that passes through $\pi\left(E_{1}\right)$ and $\pi\left(E_{2}\right)$. Then $E_{1}, E_{2}$, and $L$ are all (-1)-curves in $S$.

The pencil $\left|E_{2}+L\right|$ contains a unique curve that passes though $C \cap$ $E_{1}$. Similarly, $\left|E_{1}+L\right|$ contains a unique curve that passes though $C \cap E_{2}$. Denote these curves by $L_{1}$ and $L_{2}$, respectively. Then $L_{1}$ is irreducible and smooth unless $L_{1}=E_{2}+L$ (in this case $E_{1} \cap L \in C$ ). Similarly, the curve $L_{2}$ is irreducible and smooth unless $L_{2}=E_{1}+L$ and $L \cap E_{2} \in C$.

If $C$ does not contain the points $E_{1} \cap L$ nor $E_{2} \cap L$, then there exists a unique smooth irreducible curve $R \in\left|E_{1}+E_{2}+L\right|$ such that $R$ passes though $C \cap L$ and is tangent to $C$ at the point $C \cap L$. If either $E_{1} \cap L \in C$ or $E_{2} \cap L \in C$, we let $R=E_{1}+E_{2}+L$. In the former case, either $R$ and $C$ have simple tangency at the point $C \cap L$ or the curve $R$ is tangent to $C$ at the point $C \cap L$ with multiplicity 3 (in this case, we must have $R \cap C=C \cap L$, because $R \cdot C=3$ ). 
If either $E_{1} \cap L \in C$ or $E_{2} \cap L \in C$ (but not both, since $C \cdot L=1$ ), then we let

$$
\hat{\alpha}(S,(1-\beta) C)=\min \left\{1, \frac{1+\beta}{5 \beta}, \frac{1}{3 \beta}\right\}=\left\{\begin{array}{l}
1 \text { for } 0<\beta \leqslant \frac{1}{4}, \\
\frac{1+\beta}{5 \beta} \text { for } \frac{1}{4} \leqslant \beta \leqslant \frac{2}{3}, \\
\frac{1}{3 \beta} \text { for } \frac{2}{3} \leqslant \beta \leqslant 1 .
\end{array}\right.
$$

If the curve $C$ does not contain the points $E_{1} \cap L$ nor $E_{2} \cap L$, and either $L_{1}$ is tangent to $C$ at the point $C \cap E_{1}$ or $L_{2}$ is tangent to $C$ at the point $C \cap E_{2}$, then we let

$$
\hat{\alpha}(S,(1-\beta) C)=\min \left\{1, \frac{1+2 \beta}{6 \beta}, \frac{1}{3 \beta}\right\}=\left\{\begin{array}{l}
1 \text { for } 0<\beta \leqslant \frac{1}{4}, \\
\frac{1+2 \beta}{6 \beta} \text { for } \frac{1}{4} \leqslant \beta \leqslant \frac{1}{2}, \\
\frac{1}{3 \beta} \text { for } \frac{1}{2} \leqslant \beta \leqslant 1 .
\end{array}\right.
$$

If the curve $C$ does not contain the points $E_{1} \cap L$ nor $E_{2} \cap L$ (this implies that the curve $R$ is smooth), neither $L_{1}$ is tangent to $C$ at the point $C \cap E_{1}$ nor $L_{2}$ is tangent to $C$ at the point $C \cap E_{2}$, and the curve $R$ is tangent to $C$ at the point $C \cap L$ with multiplicity 3 , then we let

$$
\hat{\alpha}(S,(1-\beta) C)=\min \left\{1, \frac{1+3 \beta}{7 \beta}, \frac{1}{3 \beta}\right\}=\left\{\begin{array}{l}
1 \text { for } 0<\beta \leqslant \frac{1}{4}, \\
\frac{1+3 \beta}{7 \beta} \text { for } \frac{1}{4} \leqslant \beta \leqslant \frac{4}{9}, \\
\frac{1}{3 \beta} \text { for } \frac{4}{9} \leqslant \beta \leqslant 1 .
\end{array}\right.
$$

Finally, if the curve $C$ does not contain the points $E_{1} \cap L$ nor $E_{2} \cap L$ (and hence the curve $R$ is smooth), neither $L_{1}$ is tangent to $C$ at the point $C \cap E_{1}$ nor $L_{2}$ is tangent to $C$ at the point $C \cap E_{2}$, and $R$ is tangent to $C$ at the point $C \cap L$ with multiplicity 2 , then we let

$$
\hat{\alpha}(S,(1-\beta) C)=\min \left\{1, \frac{1}{3 \beta}\right\}=\left\{\begin{array}{l}
1 \text { for } 0<\beta \leqslant \frac{1}{3} \\
\frac{1}{3 \beta} \text { for } \frac{1}{3} \leqslant \beta \leqslant 1 .
\end{array}\right.
$$

We have $\hat{\alpha}(S,(1-\beta) C) \geqslant \check{\alpha}(S,(1-\beta) C)$. Indeed, if either $E_{1} \cap L \in C$ or $E_{2} \cap L \in C$, then

$$
\hat{\alpha}(S,(1-\beta) C)=\min \left\{\operatorname{lct}(S,(1-\beta) C ; \beta C), \operatorname{lct}\left(S,(1-\beta) C ; \beta\left(3 L+2 E_{1}+2 E_{2}\right)\right)\right\},
$$


which implies that $\hat{\alpha}(S,(1-\beta) C) \geqslant \check{\alpha}(S,(1-\beta) C)$. If neither $E_{1} \cap L \in C$ nor $E_{2} \cap L \in C$, then

$\min \left\{\operatorname{lct}(S,(1-\beta) C ; \beta C), \operatorname{lct}\left(S,(1-\beta) C ; \beta\left(3 L+2 E_{1}+2 E_{2}\right)\right)\right\}=\min \left\{1, \frac{1}{3 \beta}\right\}$.

If the curve $C$ does not contain the points $E_{1} \cap L$ nor $E_{2} \cap L$, and $L_{1}$ is tangent to $C$ at the point $C \cap E_{1}$, then

$$
\hat{\alpha}(S,(1-\beta) C)=\min \left\{1, \frac{1}{3 \beta}, \operatorname{lct}\left(S,(1-\beta) C ; \beta\left(2 L_{1}+2 E_{1}+L\right)\right)\right\},
$$

and similarly if $L_{2}$ is tangent to $C$ at the point $C \cap E_{2}$. If the curve $C$ does not contain the points $E_{1} \cap L$ nor $E_{2} \cap L$ (this implies that the curve $R$ is smooth), neither $L_{1}$ is tangent to $C$ at the point $C \cap E_{1}$ nor $L_{2}$ is tangent to $C$ at the point $C \cap E_{2}$, and the curve $R$ is tangent to $C$ at the point $C \cap L$ with multiplicity 3 , then

$\min \left\{\operatorname{lct}(S,(1-\beta) C ; \beta C), \operatorname{lct}\left(S,(1-\beta) C ; \beta\left(3 L+2 E_{1}+2 E_{2}\right)\right), \operatorname{lct}(S,(1-\beta) C ; \beta(L+2 R))\right\}$

equals $\hat{\alpha}(S,(1-\beta) C)$. We conclude that $\hat{\alpha}(S,(1-\beta) C) \geqslant \check{\alpha}(S,(1-\beta) C)$ in every case.

2.5. Blow up of $\mathbb{P}^{2}$ at three points. Suppose that $K_{S}^{2}=6$. Then there exists a birational morphism $\pi: S \rightarrow \mathbb{P}^{2}$ that is the blow up of three noncolinear points. Denote the $\pi$-exceptional curves by $E_{1}, E_{2}, E_{3}$, denote the proper transform on $S$ of the line in $\mathbb{P}^{2}$ that passes through $\pi\left(E_{1}\right)$ and $\pi\left(E_{2}\right)$ by $L_{12}$, denote the proper transform on $S$ of the line in $\mathbb{P}^{2}$ that passes through $\pi\left(E_{1}\right)$ and $\pi\left(E_{3}\right)$ by $L_{13}$, and denote the proper transform on $S$ of the line in $\mathbb{P}^{2}$ that passes through $\pi\left(E_{2}\right)$ and $\pi\left(E_{3}\right)$ by $L_{23}$. Then $E_{1}, E_{2}$, $E_{3}, L_{12}, L_{13}$ and $L_{23}$ are all the lines in $S$.

If the curve $C$ contains an intersection point of two intersecting lines in $S$, then we let

$$
\hat{\alpha}(S,(1-\beta) C)=\min \left\{1, \frac{1+\beta}{4 \beta}\right\}=\left\{\begin{array}{l}
1 \text { for } 0<\beta \leqslant \frac{1}{3} \\
\frac{1+\beta}{4 \beta} \text { for } \frac{1}{3} \leqslant \beta \leqslant 1 .
\end{array}\right.
$$

If the curve $C$ does not contain the intersection points of any two intersecting lines, and there are a line $Z_{1}$ and an irreducible conic $Z_{2}$ in $S$ such that $Z_{2}$ is tangent to $C$ at the point $C \cap Z_{1}$, then we let

$$
\hat{\alpha}(S,(1-\beta) C)=\min \left\{1, \frac{1+2 \beta}{5 \beta}, \frac{1}{2 \beta}\right\}=\left\{\begin{array}{l}
1 \text { for } 0<\beta \leqslant \frac{1}{3}, \\
\frac{1+2 \beta}{5 \beta} \text { for } \frac{1}{3} \leqslant \beta \leqslant \frac{3}{4}, \\
\frac{1}{2 \beta} \text { for } \frac{3}{4} \leqslant \beta \leqslant 1 .
\end{array}\right.
$$


If $C$ does not contain the intersection point of any two intersecting lines, and for every line $Z_{1}$ in $S$, there exists no irreducible conic $Z_{2}$ in $S$ such that $Z_{2}$ is tangent to $C$ at $C \cap Z_{1}$, then we let

$$
\hat{\alpha}(S,(1-\beta) C)=\min \left\{1, \frac{1}{2 \beta}\right\}=\left\{\begin{array}{l}
1 \text { for } 0<\beta \leqslant \frac{1}{2}, \\
\frac{1}{2 \beta} \text { for } \frac{1}{2} \leqslant \beta \leqslant 1 .
\end{array}\right.
$$

One has $\hat{\alpha}(S,(1-\beta) C) \geqslant \check{\alpha}(S,(1-\beta) C)$. Indeed, we have $2 E_{1}+2 L_{12}+$ $L_{13}+E_{2} \sim-K_{S}$. Thus, if $E_{1} \cap L_{12} \notin C, E_{1} \cap L_{13} \notin C$ and $E_{2} \cap L_{12} \notin C$, then

$\min \left\{\operatorname{lct}(S,(1-\beta) C ; \beta C), \operatorname{lct}\left(S,(1-\beta) C ; \beta\left(2 E_{1}+2 L_{12}+L_{13}+E_{2}\right)\right)\right\}=\left\{\begin{array}{l}1 \text { for } 0<\beta \leqslant \frac{1}{2} \\ \frac{1}{2 \beta} \text { for } \frac{1}{2} \leqslant \beta \leqslant 1\end{array}\right.$

Otherwise, this minimum is $\hat{\alpha}(S,(1-\beta) C)$. This shows that $\hat{\alpha}(S,(1-\beta) C) \geqslant$ $\check{\alpha}(S,(1-\beta) C)$ except for the case when $C$ does not contain the intersection point of any two intersecting lines, but there are a line $Z_{1}$ and a conic $Z_{2}$ in $S$ such that $Z_{2}$ is tangent to $C$ at the point $C \cap Z_{1}$. In the latter case, we may assume that $Z_{1}=E_{1}$ and $Z_{2} \in\left|L_{12}+E_{2}\right|$, which implies that

$\hat{\alpha}(S,(1-\beta) C)=\min \left\{\operatorname{lct}(S,(1-\beta) C ; \beta C), \operatorname{lct}\left(S,(1-\beta) C ; \beta\left(2 Z_{2}+E_{1}+L_{23}\right)\right)\right\}$,

since $2 Z_{2}+E_{1}+L_{23} \sim-K_{S}$. Thus, in all cases we have $\hat{\alpha}(S,(1-\beta) C) \geqslant$ $\check{\alpha}(S,(1-\beta) C)$.

2.6. Blow up of $\mathbb{P}^{2}$ at four points. Suppose that $K_{S}^{2}=5$. Then there exists a birational morphism $\pi: S \rightarrow \mathbb{P}^{2}$ that contracts four smooth rational curves to four points such that no three of them are colinear. Denote these curves by $E_{1}, E_{2}, E_{3}, E_{4}$. For and integers $i$ and $j$ such that $1 \leqslant i<j \leqslant 4$, denote by $L_{i j}$ the proper transform on $S$ via $\pi$ of the line in $\mathbb{P}^{2}$ that passes through $\pi\left(E_{i}\right)$ and $\pi\left(E_{j}\right)$. These gives us six lines $L_{12}, L_{13}, L_{14}, L_{23}, L_{24}$ and $L_{34}$. Moreover, $E_{1}, E_{2}, E_{3}, E_{4}, L_{12}, L_{13}, L_{14}, L_{23}, L_{24}$ and $L_{34}$ are all the lines in $S$. Let

$$
\hat{\alpha}(S,(1-\beta) C)=\min \left\{1, \frac{1}{2 \beta}\right\}=\left\{\begin{array}{l}
1 \text { for } 0<\beta \leqslant \frac{1}{2} \\
\frac{1}{2 \beta} \text { for } \frac{1}{2} \leqslant \beta \leqslant 1 .
\end{array}\right.
$$

Then $\hat{\alpha}(S,(1-\beta) C) \geqslant \check{\alpha}(S,(1-\beta) C)$, since $2 E_{1}+L_{12}+L_{13}+L_{14} \sim-K_{S}$ and

$\hat{\alpha}(S,(1-\beta) C)=\min \left\{\operatorname{lct}(S,(1-\beta) C ; \beta C), \operatorname{lct}\left(S,(1-\beta) C ; \beta\left(2 E_{1}+L_{12}+L_{13}+L_{14}\right)\right)\right\}$. 
2.7. Complete intersections of two quadrics. Suppose that $K_{S}^{2}=4$. Then there exists a birational morphism $\pi: S \rightarrow \mathbb{P}^{2}$ that is the blow up of five points such that no three of them are colinear. Denote by $E_{1}, E_{2}$, $E_{3}, E_{4}$ and $E_{5}$ the $\pi$-exceptional curves. For any integers $i$ and $j$ such that $1 \leqslant i<j \leqslant 5$, denote by $L_{i j}$ the proper transform via $\pi$ on $S$ of the line in $\mathbb{P}^{2}$ that passes through $\pi\left(E_{i}\right)$ and $\pi\left(E_{j}\right)$. Denote by $E$ the proper transform on $S$ of the unique smooth conic in $\mathbb{P}^{2}$ that passes through $\pi\left(E_{1}\right), \pi\left(E_{2}\right)$, $\pi\left(E_{3}\right), \pi\left(E_{4}\right)$ and $\pi\left(E_{5}\right)$. Then $E_{1}, E_{2}, E_{3}, E_{4}, E_{5}, L_{12}, L_{13}, L_{14}, L_{15}, L_{23}$, $L_{24}, L_{25}, L_{34}, L_{35}, L_{45}$ and $E$ are all the lines in $S$.

If the curve $C$ contains the intersection point of any two intersecting lines, then we let

$$
\hat{\alpha}(S,(1-\beta) C)=\min \left\{1, \frac{1+\beta}{3 \beta}\right\}=\left\{\begin{array}{l}
1 \text { for } 0<\beta \leqslant \frac{1}{2} \\
\frac{1+\beta}{3 \beta} \text { for } \frac{1}{2} \leqslant \beta \leqslant 1
\end{array}\right.
$$

If the curve $C$ does not contain the intersection point of any two intersecting lines, but there are two conics $C_{1}$ and $C_{2}$ in $S$ such that $C_{1}+C_{2} \sim-K_{S}$, and $C_{1}$ and $C_{2}$ both tangent $C$ at one point, then we let

$$
\hat{\alpha}(S,(1-\beta) C)=\min \left\{1, \frac{1+2 \beta}{4 \beta}, \frac{2}{3 \beta}\right\}=\left\{\begin{array}{l}
1 \text { for } 0<\beta \leqslant \frac{1}{2}, \\
\frac{1+2 \beta}{4 \beta} \text { for } \frac{1}{2} \leqslant \beta \leqslant \frac{5}{6}, \\
\frac{2}{3 \beta} \text { for } \frac{5}{6} \leqslant \beta \leqslant 1 .
\end{array}\right.
$$

Finally, if the curve $C$ does not contain the intersection point of any two intersecting lines, and for every two conics $C_{1}$ and $C_{2}$ in $S$ such that $C_{1}+$ $C_{2} \sim-K_{S}$, the conics $C_{1}$ and $C_{2}$ do not tangent $C$ at one point, then we let

$$
\hat{\alpha}(S,(1-\beta) C)=\min \left\{1, \frac{2}{3 \beta}\right\}=\left\{\begin{array}{l}
1 \text { for } 0<\beta \leqslant \frac{2}{3}, \\
\frac{2}{3 \beta} \text { for } \frac{2}{3} \leqslant \beta \leqslant 1 .
\end{array}\right.
$$

We claim that $\hat{\alpha}(S,(1-\beta) C) \geqslant \check{\alpha}(S,(1-\beta) C)$. Indeed, the lines $L_{12}$ and $L_{34}$ intersect at a single point. Let $Z$ be the proper transform on $S$ of the line in $\mathbb{P}^{2}$ that passes through $\pi\left(E_{5}\right)$ and $\pi\left(L_{12} \cap L_{34}\right)$. Then $L_{12}+L_{34}+Z \sim-K_{S}$. Moreover, if $L_{12} \cap L_{34} \in C$, then

$\min \left\{\operatorname{lct}(S,(1-\beta) C ; \beta C), \operatorname{lct}\left(S,(1-\beta) C ; \beta\left(L_{12}+L_{34}+Z\right)\right)\right\}=\left\{\begin{array}{l}1 \text { for } 0<\beta \leqslant \frac{1}{2}, \\ \frac{1+\beta}{3 \beta} \text { for } \frac{1}{2} \leqslant \beta \leqslant 1 .\end{array}\right.$

However, if $L_{12} \cap L_{34} \notin C$, then this minimum equals $\min \left\{1, \frac{2}{3 \beta}\right\}$. Since we can repeat these computations for any pair of intersecting lines in $S$, we see that $\hat{\alpha}(S,(1-\beta) C) \geqslant \check{\alpha}(S,(1-\beta) C)$ except possibly the case when $C$ does 
not contain the intersection point of any two intersecting lines, but there are two conics $C_{1}$ and $C_{2}$ in $S$ such that $C_{1}+C_{2} \sim-K_{S}$, and $C_{1}$ and $C_{2}$ both tangent $C$ at one point. In the latter case, $\hat{\alpha}(S,(1-\beta) C)$ is equal to $\min \left\{\operatorname{lct}(S,(1-\beta) C ; \beta C), \operatorname{lct}\left(S,(1-\beta) C ; \beta\left(L_{12}+L_{34}+Z\right)\right), \operatorname{lct}\left(S,(1-\beta) C ; \beta\left(C_{1}+C_{2}\right)\right)\right\}$, since $C_{1}+C_{2} \sim-K_{S}$. This shows that $\hat{\alpha}(S,(1-\beta) C) \geqslant \check{\alpha}(S,(1-\beta) C)$ in all three cases.

2.8. Cubic surfaces. Suppose that $K_{S}^{2}=3$. Then $S$ is a smooth cubic surface in $\mathbb{P}^{3}$. Recall that an Eckardt point in $S$ is a point of intersection of three lines contained in $S$. General cubic surface contains no Eckardt points. If $S$ contains an Eckardt point that is contained in $C$, then we let

$$
\hat{\alpha}(S,(1-\beta) C)=\min \left\{1, \frac{1+\beta}{3 \beta}\right\}=\left\{\begin{array}{l}
1 \text { for } 0<\beta \leqslant \frac{1}{2} \\
\frac{1+\beta}{3 \beta} \text { for } \frac{1}{2} \leqslant \beta \leqslant 1
\end{array}\right.
$$

If $S$ contains an Eckardt point and $C$ contains no Eckardt points, then we let

$$
\hat{\alpha}(S,(1-\beta) C)=\min \left\{1, \frac{2}{3 \beta}\right\}=\left\{\begin{array}{l}
1 \text { for } 0<\beta \leqslant \frac{2}{3}, \\
\frac{2}{3 \beta} \text { for } \frac{2}{3} \leqslant \beta \leqslant 1 .
\end{array}\right.
$$

If $S$ contains no Eckardt points, but $S$ contains a line $L$ and a conic $M$ such that $L$ is tangent to $M$ and $L \cap M \in C$, then we let

$$
\hat{\alpha}(S,(1-\beta) C)=\min \left\{1, \frac{2+\beta}{4 \beta}\right\}=\left\{\begin{array}{l}
1 \text { for } 0<\beta \leqslant \frac{2}{3}, \\
\frac{2+\beta}{4 \beta} \text { for } \frac{2}{3} \leqslant \beta \leqslant 1 .
\end{array}\right.
$$

If $S$ contains no Eckardt points, for every line $L$ and every conic $M$ on $S$ such that $L$ is tangent to $M$, we have $L \cap M \notin C$, but there is a cuspidal curve $T \in\left|-K_{S}\right|$ such that $T \cap C=\operatorname{Sing}(T)$, then we let

$$
\hat{\alpha}(S,(1-\beta) C)=\min \left\{1, \frac{2+3 \beta}{6 \beta}, \frac{3}{4 \beta}\right\}=\left\{\begin{array}{l}
1 \text { for } 0<\beta \leqslant \frac{2}{3}, \\
\frac{2+3 \beta}{6 \beta} \text { for } \frac{2}{3} \leqslant \beta \leqslant \frac{5}{6}, \\
\frac{3}{4 \beta} \text { for } \frac{5}{6} \leqslant \beta \leqslant 1 .
\end{array}\right.
$$

Finally, if $S$ contains no Eckardt points, for every line $L$ and every conic $M$ on $S$ such that $L$ is tangent to $M$ we have $L \cap M \notin C$, and every irreducible 
cuspidal curve $T \in\left|-K_{S}\right|$ intersects $C$ by at least two point, then we let

$$
\hat{\alpha}(S,(1-\beta) C)=\min \left\{1, \frac{3}{4 \beta}\right\}=\left\{\begin{array}{l}
1 \text { for } 0<\beta \leqslant \frac{3}{4}, \\
\frac{3}{4 \beta} \text { for } \frac{3}{4} \leqslant \beta \leqslant 1 .
\end{array}\right.
$$

One can easily check that $\hat{\alpha}(S,(1-\beta) C) \geqslant \check{\alpha}(S,(1-\beta) C)$ (see $[13$, Theorem 4.9.1]).

2.9. Double covers of $\mathbb{P}^{2}$. Suppose that $K_{S}^{2}=2$. Recall that $C$ is a nonsingular curve in the linear system $\left|-K_{S}\right|$. Different choices of $C$ will give rise to different invariants $\hat{\alpha}(S,(1-\beta) C)$ whose values depend on the existence of certain singular irreducible curves $Z \in\left|-K_{S}\right|$ such that $C$ contains the singular point of $Z$. We give the different values of $\hat{\alpha}(S,(1-\beta) C)$ for all smooth $C \in\left|-K_{S}\right|$ distinguishing four possible cases for the curve $Z$.

If $\left|-K_{S}\right|$ contains a tacnodal curve $Z$ whose singular point is contained in $C$, then we let

$$
\hat{\alpha}(S,(1-\beta) C)=\min \left\{1, \frac{2+\beta}{4 \beta}\right\}=\left\{\begin{array}{l}
1 \text { for } 0<\beta \leqslant \frac{2}{3}, \\
\frac{2+\beta}{4 \beta} \text { for } \frac{2}{3} \leqslant \beta \leqslant 1 .
\end{array}\right.
$$

If $\left|-K_{S}\right|$ contains at least one tacnodal curve $Z$, but $C$ does not contain singular points of any tacnodal curve in $\left|-K_{S}\right|$, then we let

$$
\hat{\alpha}(S,(1-\beta) C)=\min \left\{1, \frac{3}{4 \beta}\right\}=\left\{\begin{array}{l}
1 \text { for } 0<\beta \leqslant \frac{3}{4}, \\
\frac{3}{4 \beta} \text { for } \frac{3}{4} \leqslant \beta \leqslant 1 .
\end{array}\right.
$$

If $\left|-K_{S}\right|$ contains no curves with tacnodal singularities but there is at least one cuspidal rational point $Z \in\left|-K_{S}\right|$ such that $C$ contains the cuspidal singular point of $Z$, then we let

$$
\hat{\alpha}(S,(1-\beta) C)=\min \left\{1, \frac{3+2 \beta}{6 \beta}\right\}=\left\{\begin{array}{l}
1 \text { for } 0<\beta \leqslant \frac{3}{4} \\
\frac{3+2 \beta}{6 \beta} \text { for } \frac{3}{4} \leqslant \beta \leqslant 1 .
\end{array}\right.
$$

Finally, if $\left|-K_{S}\right|$ contains no curves with tacnodal singularities, and $C$ does not contain the cuspidal singular point of any cuspidal rational curves in $\left|-K_{S}\right|$, then we let

$$
\hat{\alpha}(S,(1-\beta) C)=\min \left\{1, \frac{5}{6 \beta}\right\}=\left\{\begin{array}{l}
1 \text { for } 0<\beta \leqslant \frac{5}{6}, \\
\frac{5}{6 \beta} \text { for } \frac{5}{6} \leqslant \beta \leqslant 1 .
\end{array}\right.
$$


Clearly, this four cases exhaust all possibilities for smooth curves $C \in$ $\left|-K_{S}\right|$. One can easily check that $\hat{\alpha}(S,(1-\beta) C) \geqslant \check{\alpha}(S,(1-\beta) C)$ (see [13, Theorem 4.10.1]).

2.10. Double covers of quadric cones. Suppose that $K_{S}^{2}=1$. As above, $C$ is a non-singular curve in the pencil $\left|-K_{S}\right|$. If $\left|-K_{S}\right|$ contains no cuspidal curves, then we let $\hat{\alpha}(S,(1-\beta) C)=1$ for every $\beta \in(0,1]$. Otherwise, we let

$$
\hat{\alpha}(S,(1-\beta) C)=\min \left\{1, \frac{5}{6 \beta}\right\}=\left\{\begin{array}{l}
1 \text { for } 0<\beta \leqslant \frac{5}{6} \\
\frac{5}{6 \beta} \text { for } \frac{5}{6} \leqslant \beta \leqslant 1 .
\end{array}\right.
$$

In the former case, we have $\hat{\alpha}(S,(1-\beta) C)=\operatorname{lct}(S,(1-\beta) C ; \beta C)$. In the latter case, we have

$$
\hat{\alpha}(S,(1-\beta) C)=\min \{\operatorname{lct}(S,(1-\beta) C ; \beta C), \operatorname{lct}(S,(1-\beta) C ; \beta Z)\},
$$

where $Z$ is a cuspidal curve in $\left|-K_{S}\right|$. Thus, $\hat{\alpha}(S,(1-\beta) C) \geqslant \check{\alpha}(S,(1-\beta) C)$ in both cases.

\section{Local Inequalities}

Let $S$ be a smooth surface, let $D$ be an effective $\mathbb{R}$-divisor on $S$, and let $P$ be a point in $S$.

Lemma 3.1. Suppose that $(S, D)$ is not log canonical at $P$. Then $\operatorname{mult}_{P}(D)>$ 1.

Proof. This is a well-known fact. See [8, Exercise 6.18], for instance.

Lemma 3.2. Suppose that $(S, D)$ is not $\log$ canonical at $P$. Let $B$ be an effective $\mathbb{R}$-divisor on $S$ such that $(S, B)$ is $\log$ canonical and $B \sim_{\mathbb{R}} D$. Then there exists an effective $\mathbb{R}$-divisor $D^{\prime}$ on $S$ such that $D^{\prime} \sim_{\mathbb{R}} D$, the log pair $\left(S, D^{\prime}\right)$ is not $\log$ canonical at $P$, and $\operatorname{Supp}\left(D^{\prime}\right)$ does not contain at least one irreducible component of $\operatorname{Supp}(B)$.

Proof. Let $\mu$ be the greatest real number such that $D^{\prime}:=(1+\mu) D-\mu B$ is effective. Since $D \neq B$, the number $\mu$ does exist. Then $D^{\prime} \sim_{\mathbb{R}} D$, the $\log$ pair $\left(S, D^{\prime}\right)$ is not $\log$ canonical at $P$, and $\operatorname{Supp}\left(D^{\prime}\right)$ does not contain at least one irreducible component of $\operatorname{Supp}(B)$.

Let $\pi_{1}: S_{1} \rightarrow S$ be a blow up of the point $P$, let $F_{1}$ be the $\pi$-exceptional curve, and let $D^{1}$ be the proper transform of $D$ via $\pi_{1}$. Then $K_{S_{1}}+D^{1}+$ $\left(\operatorname{mult}_{P}(D)-1\right) F_{1} \sim_{\mathbb{R}} \pi_{1}^{*}\left(K_{S}+D\right)$.

Lemma 3.3. Suppose that $(S, D)$ is not $\log$ canonical at $P$. Then $\operatorname{mult}_{P}(D)>$ 1 and there exists a point $P_{1} \in F_{1}$ such that $\left(S_{1}, D^{1}+\left(\operatorname{mult}_{P}(D)-1\right) F_{1}\right)$ is not log canonical at $P_{1}$. Moreover, one has $\operatorname{mult}_{P}(D)+\operatorname{mult}_{P_{1}}\left(D^{1}\right)>2$. If, in addition, $\operatorname{mult}_{P}(D) \leqslant 2$, then such point $P_{1}$ is unique.

Proof. This is a well-known fact. See, for example, [3, Remark 2.5]. 
Let $C$ be an irreducible curve on $S$ that contains $P$. Suppose that $C$ is smooth at $P$. Write $D=a C+\Omega$, where $a \in \mathbb{R}_{\geqslant 0}$, and $\Omega$ is an effective $\mathbb{R}$-divisor on $S$ with $C \not \subset \operatorname{Supp}(\Omega)$.

Theorem 3.4. If $(S, a C+\Omega)$ is not $\log$ canonical at $P$ and $a \leqslant 1$, then $\operatorname{mult}_{P}(\Omega \cdot C)>1$.

Proof. See, for example, [8, Exercise 6.31], [14, Lemma 2.5] or [2, Theorem 7].

Denote the proper transform of the curve $C$ on the surface $S_{1}$ by $C^{1}$, and denote the proper transform of the $\mathbb{R}$-divisor $\Omega$ on the surface $S_{1}$ by $\Omega^{1}$.

Lemma 3.5. Suppose that $a \leqslant 1$, the log pair $(S, a C+\Omega)$ is not log canonical at the point $P$, and $\operatorname{mult}_{P}(\Omega) \leqslant 1$. Then $\left(S_{1}, a C^{1}+\Omega^{1}+\left(a+\operatorname{mult}_{P}(\Omega)-\right.\right.$ 1) $F_{1}$ ) is not log canonical at $C^{1} \cap F_{1}$, it is log canonical at every point in $E_{1} \backslash\left(C^{1} \cap F_{1}\right)$, and $\operatorname{mult}_{P}(\Omega \cdot C)>2-a$.

Proof. Since $a \leqslant 1$ and $\operatorname{mult}_{P}(\Omega) \leqslant 1$, we have $\operatorname{mult}_{P}(D) \leqslant 2$. By Lemma 3.3, there exists a unique point $P_{1} \in F_{1}$ such that the $\log$ pair $\left(S_{1}, a C^{1}+\Omega^{1}+\left(a+\operatorname{mult}_{P}(\Omega)-1\right) F_{1}\right)$ is not $\log$ canonical at $P_{1}$. If $P_{1} \notin C^{1}$, then $\operatorname{mult}_{P}(\Omega)=F_{1} \cdot \Omega^{1} \geqslant \operatorname{mult}_{P_{1}}\left(\Omega^{1} \cdot F_{1}\right)>1$ by Theorem 3.4, which is impossible, since $\operatorname{mult}_{P}(\Omega) \leqslant 1$. Thus, $P_{1} \in C^{1}$. Then, by Theorem 3.4 again:

$$
\operatorname{mult}_{P}(\Omega \cdot C) \geqslant \operatorname{mult}_{P}(\Omega)+\operatorname{mult}_{P_{1}}\left(\Omega^{1} \cdot C^{1}\right)>2-a .
$$

Let us consider an infinite sequence of blow ups

$$
\cdots \stackrel{\pi_{n+1}}{\longrightarrow} S_{n} \stackrel{\pi_{n}}{\longrightarrow} S_{n-1} \stackrel{\pi_{n-1}}{\longrightarrow} \cdots \stackrel{\pi_{3}}{\longrightarrow} S_{2} \stackrel{\pi_{2}}{\longrightarrow} S_{1} \stackrel{\pi_{1}}{\longrightarrow} S
$$

such that each $\pi_{n}$ is the blow up of the point in the proper transform of the curve $C$ on the surface $S_{n-1}$ that dominates $P$. Denote the $\pi_{n}$-exceptional curve by $F_{n}$, and denote the proper transform of $C$ on $S_{n}$ by $C^{n}$. For every $n \geqslant 1$, write $P_{n}=C^{n} \cap F_{n}$, denote the proper transform of the divisor $\Omega$ on $S_{n}$ by $\Omega^{n}$, let $m_{n}=\operatorname{mult}_{P_{n}}\left(\Omega^{n}\right)$ and let $m_{0}=\operatorname{mult}_{P}(\Omega)$. For every positive integers $k \leqslant n$, denote the proper transform of the curve $F_{k}$ on $S_{n}$ by $F_{k}^{n}$. Finally, we let

$$
D^{S_{n}}=a C^{n}+\Omega^{n}+\sum_{k=1}^{n}\left(k a-k+\sum_{i=0}^{k-1} m_{i}\right) F_{k}^{n}
$$

for every $n \geqslant 1$. Then $K_{S_{n}}+D^{S_{n}} \sim_{\mathbb{R}}\left(\pi_{1} \circ \pi_{2} \circ \cdots \circ \pi_{n}\right)^{*}\left(K_{S}+D\right)$ for every $n \geqslant 1$.

Theorem 3.6. Suppose that $(S, a C+\Omega)$ is not $\log$ canonical at $P$ and $a \leqslant 1$. Then $m_{0}+a>1$ and $\operatorname{mult}_{P}(\Omega \cdot C)>1$. Moreover, the following additional assertions hold:

(i) if $m_{0} \leqslant 1$, then the log pair $\left(S_{1}, D^{S_{1}}\right)$ is not log canonical at $P_{1}$, 
(ii) if $\left(S_{n}, D^{S_{n}}\right)$ is not log canonical at some point in $F_{n}$, then $D^{S_{n}}$ is an effective divisor,

(iii) if $\left(S_{n}, D^{S_{n}}\right)$ is not log canonical at some point in $F_{n}$ and $\sum_{i=0}^{n-1} m_{i} \leqslant$ $n+1-n a$, then such point in $F_{n}$ is unique,

(iv) if $\left(S_{n}, D^{S_{n}}\right)$ is not log canonical at $P_{n}$, then $(n+1) a+\sum_{i=0}^{n} m_{i}>n+2$, the log pair $\left(S_{n+1}, D^{S_{n+1}}\right)$ is not log canonical at some point in $F_{n+1}$, and $\operatorname{mult}_{P}(\Omega \cdot C)>n+1-n a$,

(v) if $n \geqslant 2, m_{n-1} \leqslant 1$ and $\sum_{i=0}^{n-1} m_{i} \leqslant n+1-n a$, then $\left(S_{n}, D^{S_{n}}\right)$ is $\log$ canonical at every point of $F_{n}$ different from $P_{n}$ and $F_{n} \cap F_{n-1}^{n}$,

(vi) if $n \geqslant 2$ and $\sum_{i=0}^{n-1} m_{i} \leqslant n-(n-1) a$, then $\left(S_{n}, D^{S_{n}}\right)$ is log canonical at $F_{n} \cap F_{n-1}^{n}$,

(vii) if $n \geqslant 2, \sum_{i=0}^{n-2} m_{i} \leqslant n-(n-1) a$, and $\sum_{i=0}^{n-3} m_{i}+2 m_{n-2} \leqslant n+1-n a$, then $\left(S_{n}, D^{S_{n}}\right)$ is log canonical at $F_{n} \cap F_{n-1}^{n}$.

Proof. By Lemma 3.1, we have $m_{0}+a>1$. By Theorem 3.4, we have $\operatorname{mult}_{P}(\Omega \cdot C)>1-a$. Assertion (i) follows from Lemma 3.5.

For assertion (ii), it is enough to show that $k(a-1)+\sum_{i=0}^{k-1} m_{i} \geqslant 0$ for $1 \leqslant k \leqslant n$. If $a \geqslant 1$, this is clear. If $0 \leqslant a \leqslant 1$, then $m_{0}>1$ by Lemma 3.1 and hence $D^{S_{1}}$ is effective. Assertion (ii) follows by induction. Indeed, since $\left(S_{n}, D^{S_{n}}\right)$ is not $\log$ canonical at some point in $F_{n}$, then $\left(S_{l-1}, D^{S_{l-1}}\right)$ is not $\log$ canonical at $P_{l-1}$ for $1 \leqslant l \leqslant n$. If $(l-1)(a-1)+\sum_{i=0}^{l-2} m_{i} \geqslant 0$, then

$$
l(a-1)+\sum_{i-0}^{l-1} m_{i} \geqslant(a-1)+m_{l-1} \geqslant 0 .
$$

For the last inequality, we notice that the induction hypothesis gives us that $D_{k}$ is effective and $\left(S_{k}, D^{S_{k}}\right)$ is not $\log$ canonical for $0 \leqslant k \leqslant l-1$. Hence Lemma 3.1 implies that $m_{l-1}>1$, proving assertion (ii).

Inequality $\sum_{i=0}^{n-1} m_{i} \leqslant n+1-n a$ is equivalent to $\operatorname{mult}_{P_{n-1}}\left(D^{S_{n-1}}\right) \leqslant$ 2. Thus, assertion (iii) follows from Lemma 3.3. If $\left(S_{n}, D^{S_{n}}\right)$ is not $\log$ canonical at $P_{n}$, then $(n+1) a+\sum_{i=0}^{n} m_{i}>n+2$ by Lemma 3.1 , the pair $\left(S_{n+1}, D^{S_{n+1}}\right)$ is not log canonical at some point in $F_{n+1}$ by Lemma 3.3 , and

$$
\operatorname{mult}_{P}(\Omega \cdot C)-\sum_{i=0}^{n-1} m_{i}=\operatorname{mult}_{P_{n}}\left(\Omega^{n} \cdot C^{n}\right)>1-\left(n a-n+\sum_{i=0}^{n-1} m_{i}\right),
$$

by Theorem 3.4. This proves assertion (iv).

Suppose that $n \geqslant 2$. Let $O=F_{n} \cap F_{n-1}^{n}$. If $\sum_{i=0}^{n-1} m_{i} \leqslant n+1-n a$ and $\left(S_{n}, D^{S_{n}}\right)$ is not $\log$ canonical at some point in $F_{n} \backslash\left(P_{n} \cup O\right)$, then $m_{n-1}=F_{n} \cdot \Omega^{n}>1$ by Theorem 3.4 , which implies assertion (v). If $\left(S_{n}, D^{S_{n}}\right)$ is not $\log$ canonical at $O$ and $\sum_{i=0}^{n-1} m_{i} \leqslant n+1-n a$, then

$$
m_{n-1}=F_{n} \cdot \Omega^{n} \geqslant \operatorname{mult}_{O}\left(F_{n} \cdot \Omega^{n}\right)>1-\left((n-1) a-n+1+\sum_{i=0}^{n-2} m_{i}\right)
$$


by Theorem 3.4. If $\left(S_{n}, D^{S_{n}}\right)$ is not $\log$ canonical at $O$ and $\sum_{i=0}^{n-2} m_{i} \leqslant$ $n-(n-1) a$, then

$$
m_{n-2}-m_{n-1}=F_{n-1}^{n} \cdot \Omega^{n} \geqslant \operatorname{mult}_{O}\left(F_{n-1}^{n} \cdot \Omega^{n}\right)>1-\left(n a-n+\sum_{i=0}^{n-1} m_{i}\right)
$$

by Theorem 3.4. This proves assertions (vi) and (vii).

Corollary 3.7. Suppose that $(S, a C+\Omega)$ is not $\log$ canonical at $P, C \not \subset$ $\operatorname{Supp}(\Omega), a \leqslant 1$ and $m_{0} \leqslant \min \left\{1,1+\frac{1}{n}-n a\right\}$ for some integer $n \geqslant 1$. Then $\operatorname{mult}_{P}(\Omega \cdot C)>n+1-n a$.

Corollary 3.8. Suppose that $(S, a C+\Omega)$ is not log canonical at $P, a \leqslant 1$ and $m_{0} \leqslant 1$. Suppose that $2 m_{0} \leqslant 3-2 a$ or $m_{0}+m_{1} \leqslant 2-a$. Suppose that $m_{0}+2 m_{1} \leqslant 4-3 a$ or $m_{0}+m_{1}+m_{2} \leqslant 3-2 a$. Then $\operatorname{mult}_{P}(\Omega \cdot C)>$ $4-3 a$. If $m_{0}+m_{1}+2 m_{2} \leqslant 5-4 a$ or $m_{0}+m_{1}+m_{2}+m_{3} \leqslant 4-3 a$, then $\operatorname{mult}_{P}(\Omega \cdot C)>5-4 a$.

Let us conclude this section by recalling

Theorem 3.9 ([2, Theorem 13]). Let $C_{1}$ and $C_{2}$ be two irreducible curves on $S$ that are both smooth at $P$ and intersect transversally at $P$. Let $D=$ $a_{1} C_{1}+a_{2} C_{2}+\Delta$, where $a_{1}$ and $a_{2}$ are non-negative real numbers, and $\Delta$ is an effective $\mathbb{R}$-divisor on $S$ whose support does not contain the curves $C_{1}$ and $C_{2}$. If $(S, D)$ is not $\log$ canonical at $P$ and $\operatorname{mult}_{P}(\Delta) \leqslant 1$, then $\operatorname{mult}_{P}\left(\Delta \cdot C_{1}\right)>2\left(1-a_{2}\right)$ or $\operatorname{mult}_{P}\left(\Delta \cdot C_{2}\right)>2\left(1-a_{1}\right)$.

\section{The Proof}

Let us use the notation of Section 2. The goal of this section is to prove

Theorem 4.1. One has $\alpha(S,(1-\beta) C)=\hat{\alpha}(S,(1-\beta) C)$ for every $\beta \in(0,1]$.

This theorem implies Theorem 1.8, since $\hat{\alpha}(S,(1-\beta) C) \geqslant \check{\alpha}(S,(1-\beta) C)$ (see Section 2$)$ and $\check{\alpha}(S,(1-\beta) C) \geqslant \alpha(S,(1-\beta) C)$ (by definition) for every $\beta \in(0,1]$.

Let $D$ be any effective $\mathbb{R}$-divisor such that $D \sim_{\mathbb{R}}-K_{S}$, and let $P$ be any point in $S$. Since $\alpha(S,(1-\beta) C) \leqslant \hat{\alpha}(S,(1-\beta) C)$, to prove Theorem 4.1, it is enough to show that the log pair

$$
(S,(1-\beta) C+\hat{\alpha}(S,(1-\beta) C) \beta D)
$$

is $\log$ canonical at $P$ for every $\beta \in(0,1]$. We will do this in several steps.

Lemma 4.2. Suppose that (4.1) is not $\log$ canonical at $P$. Then $P \in C$, we have

$$
\operatorname{mult}_{P}(D)>\frac{1}{\hat{\alpha}(S,(1-\beta) C)} \geqslant 1
$$

and (4.1) is log canonical outside of the point $P$. Moreover, if there exists $a(-1)$-curve $Z \subset S$ such that $P \in Z$, then $Z \subset \operatorname{Supp}(D)$. Furthermore, there exists an effective $\mathbb{R}$-divisor $D^{\prime} \sim_{\mathbb{R}} D$ such that $C \not \subset \operatorname{Supp}\left(D^{\prime}\right)$ and $\left(S,(1-\beta) C+\hat{\alpha}(S,(1-\beta) C) \beta D^{\prime}\right)$ is not log canonical at $P$. 
Proof. If $P \notin C$, then $(S, \hat{\alpha}(S,(1-\beta) C) \beta D)$ is not $\log$ canonical at $P$, which is impossible, since $\alpha(S) \leqslant \beta \hat{\alpha}(S,(1-\beta) C)$ by [1, Theorem 1.7]. We have $\hat{\alpha}(S,(1-\beta) C) \operatorname{mult}_{P}(D)>1$ by Lemma 3.1. In particular, if there exists a (-1)-curve $Z \subset S$ such that $P \in Z$, then $Z$ must be contained in $\operatorname{Supp}(D)$, because otherwise we would have $1=Z \cdot D \geqslant \operatorname{mult}_{P}(D)>1$.

We see that (4.1) is log canonical outside of the curve $C$. Moreover, the coefficient of the curve $C$ in the divisor $(1-\beta) C+\hat{\alpha}(S,(1-\beta) C) \beta D$ does not exceed 1 , since $D \sim_{\mathbb{R}} C$. Hence, the $\log$ pair (4.1) is log canonical outside of finitely many points. Now the connectedness principle (see, for example, [8, Theorem 6.32]) implies that (4.1) is log canonical outside of $P$.

Since $(S,(1-\beta) C+\hat{\alpha}(S,(1-\beta) C) \beta C)$ is $\log$ canonical, it follows from Lemma 3.2 that there is an effective $\mathbb{R}$-divisor $D^{\prime} \sim_{\mathbb{R}} D$ such that $C \not \subset$ $\operatorname{Supp}\left(D^{\prime}\right)$ and $\left(S,(1-\beta) C+\hat{\alpha}(S,(1-\beta) C) \beta D^{\prime}\right)$ is not $\log$ canonical at $P$.

Thus, to prove that (4.1) is $\log$ canonical at $P$, we may assume that $P \in C \not \subset \operatorname{Supp}(D)$.

Lemma 4.3. If $S \cong \mathbb{P}^{2}$, then (4.1) is log canonical at $P$.

Proof. Suppose (4.1) is not log canonical at $P$. Let $L$ be a general line in $S$ that contains $P$. Then $\operatorname{mult}_{P}(D) \leqslant D \cdot L=3$. But $3 \hat{\alpha}(S,(1-\beta) C) \beta \leqslant \frac{1}{3}+\beta$ (see $\S 2.1$ ). Thus, if $\beta \leqslant \frac{2}{3}$, then

$$
\hat{\alpha}(S,(1-\beta) C) \beta \operatorname{mult}_{P}(D) \leqslant 3 \hat{\alpha}(S,(1-\beta) C) \beta \leqslant \frac{1}{3}+\beta \leqslant 1 .
$$

Similarly, if $\frac{2}{3} \leqslant \beta \leqslant 1$, then $\hat{\alpha}(S,(1-\beta) C) \beta \operatorname{mult}_{P}(D) \leqslant \frac{1}{3} \operatorname{mult}_{P}(D) \leqslant 1$. Applying Corollary 3.7 with $n=3$ to (4.1), we get

$9 \beta \hat{\alpha}(S,(1-\beta) C)=\hat{\alpha}(S,(1-\beta) C) \beta(C \cdot D) \geqslant \hat{\alpha}(S,(1-\beta) C) \beta \operatorname{mult}_{P}(C \cdot D)>1+3 \beta$,

which contradicts the definition of $\hat{\alpha}(S,(1-\beta) C)$ in $\S 2.1$.

Lemma 4.4. Suppose that $S \cong \mathbb{P}^{1} \times \mathbb{P}^{1}$. Then (4.1) is log canonical at $P$.

Proof. Suppose that (4.1) is not $\log$ canonical at $P$. Let $L_{1}$ and $L_{2}$ be the fibers of two different projections $S \rightarrow \mathbb{P}^{1}$ that both pass through $P$. Since $\left(S,(1-\beta) C+\hat{\alpha}(S,(1-\beta) C) \beta\left(2 L_{1}+2 L_{2}\right)\right)$ is $\log$ canonical and $2 L_{1}+$ $2 L_{2} \sim_{\mathbb{R}} D$, we may assume that either $L_{1} \not \subset \operatorname{Supp}(D)$ or $L_{2} \not \subset \operatorname{Supp}(D)$ by Lemma 3.2. This implies that $\operatorname{mult}_{P}(D) \leqslant 2$, since $D \cdot L_{1}=D \cdot L_{2}=2$. Then

$$
\hat{\alpha}(S,(1-\beta) C) \beta \operatorname{mult}_{P}(D) \leqslant 2 \hat{\alpha}(S,(1-\beta) C) \beta \leqslant \min \left\{1, \frac{1}{4}+\beta\right\},
$$

(see $\S 2.2)$. Applying Corollary 3.7 with $n=4$, we get

$8 \hat{\alpha}(S,(1-\beta) C) \beta=\hat{\alpha}(S,(1-\beta) C) \beta(C \cdot D) \geqslant \hat{\alpha}(S,(1-\beta) C) \beta \operatorname{mult}_{P}(C \cdot D)>1+4 \beta$, which contradicts the definition of $\hat{\alpha}(S,(1-\beta) C)$ in $\S 2.2$.

Lemma 4.5. Suppose that $K_{S}^{2} \leqslant 3$. Then (4.1) is log canonical at $P$. 
Proof. Suppose that (4.1) is not log canonical at P. By [3, Theorem 1.12], there is $T \in\left|-K_{S}\right|$ such that $(S, T)$ is not log canonical at $P$, and all irreducible components of the curve $T$ are contained in the support of the divisor $D$. Moreover, such $T$ is unique.

Since $(S, T)$ is not $\log$ canonical at $P$, we have very limited number of choices for $T \in\left|-K_{S}\right|$. Going through all of them, we see that $(S,(1-$ $\beta) C+\hat{\alpha}(S,(1-\beta) C) \beta T)$ is log canonical at $P$ (for details, see the proofs of $[13$, Theorems 4.9.1, 4.10.1, 4.11.1]).

By Lemma 3.2, there is an effective $\mathbb{R}$-divisor $D^{\prime}$ on the surface $S$ such that $D^{\prime} \sim_{\mathbb{R}} D$, the log pair $\left(S,(1-\beta) C+\hat{\alpha}(S,(1-\beta) C) \beta D^{\prime}\right)$ is not log canonical at $P$, and $\operatorname{Supp}\left(D^{\prime}\right)$ does not contain at least one irreducible component of $T$. The latter contradicts [3, Theorem 1.12].

Corollary 4.6. Theorem 4.1 holds in the following cases: $S \cong \mathbb{P}^{2}, S \cong$ $\mathbb{P}^{1} \times \mathbb{P}^{1}$ and $K_{S}^{2} \leqslant 3$.

Lemma 4.7. Suppose that $4 \leqslant K_{S}^{2} \leqslant 7$, and $P$ is the intersection point of two intersecting $(-1)$-curves in $S$. Then (4.1) is log canonical at $P$.

Proof. Suppose that (4.1) is not $\log$ canonical at $P$. Denote by $Z_{1}$ and $Z_{2}$ the two $(-1)$-curves in $S$ that contain $P$. We write $D=a Z_{1}+b Z_{2}+\Omega$, where $a$ and $b$ are non-negative real numbers, and $\Omega$ is an effective $\mathbb{R}$-divisor whose support does not contain $Z_{1}$ and $Z_{2}$. By Lemma 4.2, one has $a>0$ and $b>0$. Let $x=\operatorname{mult}_{P}(\Omega)$. Then $1-b+a=\Omega \cdot Z_{1} \geqslant x$, which gives $b-a+x \leqslant 1$. Similarly, we obtain $a-b+x \leqslant 1$. Then $a \leqslant 1+b, b \leqslant 1+a$ and $x \leqslant 1$. Thus, we have

$\operatorname{mult}_{P}((1-\beta) C+\hat{\alpha}(S,(1-\beta) C) \beta \Omega)=1-\beta+\hat{\alpha}(S,(1-\beta) C) \beta x \leqslant 1-\beta+\hat{\alpha}(S,(1-\beta) C) \beta \leqslant 1$,

because $\hat{\alpha}(S,(1-\beta) C) \leqslant 1$. Applying Theorem 3.9 to (4.1), we see that

$2(1-\hat{\alpha}(S,(1-\beta) C) \beta a)<Z_{1} \cdot(\hat{\alpha}(S,(1-\beta) C) \beta \Omega+(1-\beta) C)=\hat{\alpha}(S,(1-\beta) C) \beta(1-a+b)+1-\beta$, or

$2(1-\hat{\alpha}(S,(1-\beta) C) \beta b)<Z_{2} \cdot(\hat{\alpha}(S,(1-\beta) C) \beta \Omega+(1-\beta) C)=\hat{\alpha}(S,(1-\beta) C) \beta(1-b+a)+1-\beta$.

In both cases, we obtain $\hat{\alpha}(S,(1-\beta) C) \beta(1+a+b)>1+\beta$.

Suppose that $K_{S}^{2}=7$. Let us use the notation of $\S 2.4$. We may assume that $Z_{1}=E_{1}$ and $Z_{2}=L$. Since $3 L+2 E_{1}+2 E_{2} \sim-K_{S}$ and $(S,(1-\beta) C+$ $\left.\hat{\alpha}(S,(1-\beta) C) \beta\left(3 L+2 E_{1}+2 E_{2}\right)\right)$ is $\log$ canonical, we may also assume that $E_{2} \not \subset \operatorname{Supp}(\Omega)$ by Lemma 3.2. Then $1-b=E_{2} \cdot \Omega \geqslant 0$, which gives $b \leqslant 1$. Since $a \leqslant 1+b$, we get $a+b \leqslant 3$. Thus, we have

$$
4 \beta \hat{\alpha}(S,(1-\beta) C) \geqslant \hat{\alpha}(S,(1-\beta) C) \beta(1+a+b)>1+\beta,
$$

which contradicts the definition of $\hat{\alpha}(S,(1-\beta) C)$.

Suppose that $K_{S}^{2}=6$. Let us use the notation of $\S 2.5$. Without loss of generality, we may assume that $Z_{1}=E_{1}$ and $Z_{2}=L_{12}$. Since $(S,(1-\beta) C+$ $\left.\hat{\alpha}(S,(1-\beta) C) \beta\left(2 L_{12}+2 E_{1}+L_{13}+E_{2}\right)\right)$ is $\log$ canonical and $2 L_{12}+2 E_{1}+$ 
$L_{13}+E_{2} \sim-K_{S}$, we may assume that $\operatorname{Supp}(\Omega)$ does not contain $L_{13}$ or $E_{2}$ by Lemma 3.2. If $L_{13} \not \subset \operatorname{Supp}(\Omega)$, then $1-a=\Omega \cdot L_{13} \geqslant 0$, which implies that $a \leqslant 1$. Similarly, if $E_{2} \not \subset \operatorname{Supp}(\Omega)$, then $b \leqslant 1$. Since $a \leqslant 1+b$ and $b \leqslant 1+a$, we see that $a+b \leqslant 3$. Thus, we have

$$
4 \beta \hat{\alpha}(S,(1-\beta) C) \geqslant \hat{\alpha}(S,(1-\beta) C) \beta(1+a+b)>1+\beta,
$$

which contradicts the definition of $\hat{\alpha}(S,(1-\beta) C)$.

Suppose that $K_{S}^{2}=5$. Let us use the notation of $\S 2.6$. Without loss of generality, we may assume that $Z_{1}=E_{1}$ and $Z_{2}=L_{12}$. Since $(S,(1-\beta) C+$ $\left.\hat{\alpha}(S,(1-\beta) C) \beta\left(2 E_{1}+L_{12}+L_{13}+L_{14}\right)\right)$ is $\log$ canonical and $2 E_{1}+L_{12}+$ $L_{13}+L_{14} \sim-K_{S}$, we may assume that $\operatorname{Supp}(\Omega)$ does not contain $L_{13}$ or $L_{14}$ by Lemma 3.2. Since $\left(S,(1-\beta) C+\hat{\alpha}(S,(1-\beta) C) \beta\left(E_{1}+2 L_{12}+E_{2}+L_{34}\right)\right)$ is $\log$ canonical and $E_{1}+2 L_{12}+E_{2}+L_{34} \sim-K_{S}$, we may assume that $\operatorname{Supp}(\Omega)$ does not contain $E_{2}$ or $L_{34}$ by Lemma 3.2. If $L_{13} \not \subset \operatorname{Supp}(\Omega)$, then $1-a=\Omega \cdot L_{13} \geqslant 0$, which gives $a \leqslant 1$. Similarly, if $L_{14} \not \subset \operatorname{Supp}(\Omega)$, then $a \leqslant 1$. If $E_{2} \not \subset \operatorname{Supp}(\Omega)$, then $1-b=\Omega \cdot E_{2} \geqslant 0$, which gives $b \leqslant 1$. Similarly, if $L_{34} \not \subset \operatorname{Supp}(\Omega)$, then $b \leqslant 1$. Thus, we have $a \leqslant 1$ and $b \leqslant 1$. Then

$$
3 \beta \hat{\alpha}(S,(1-\beta) C) \geqslant \hat{\alpha}(S,(1-\beta) C) \beta(1+a+b)>1+\beta,
$$

which contradicts the definition of $\hat{\alpha}(S,(1-\beta) C)$.

We have $K_{S}^{2}=4$. Let us use the notation of $\S 2.7$. Without loss of generality, we may assume that $Z_{1}=L_{12}$ and $Z_{2}=L_{34}$. Let $Z$ be the proper transform on $S$ of the line in $\mathbb{P}^{2}$ that passes through $\pi\left(E_{5}\right)$ and $\pi\left(L_{12} \cap L_{34}\right)$. Since $\left(S,(1-\beta) C+\hat{\alpha}(S,(1-\beta) C) \beta\left(L_{12}+L_{34}+Z\right)\right)$ is $\log$ canonical and $L_{12}+L_{34}+Z \sim-K_{S}$, we may assume that $Z \not \subset \operatorname{Supp}(\Omega)$ by Lemma 3.2. Then $2-a-b=\Omega \cdot Z \geqslant 0$, which implies that $3 \beta \hat{\alpha}(S,(1-\beta) C) \geqslant$ $\hat{\alpha}(S,(1-\beta) C) \beta(1+a+b)>1+\beta$. The latter contradicts the definition of $\hat{\alpha}(S,(1-\beta) C)$.

Lemma 4.8. Suppose $S \cong \mathbb{F}_{1}$, and $P$ is contained in the unique $(-1)$-curve in $S$. Then (4.1) is log canonical at $P$.

Proof. Let us use the notation of $\S 2.3$. Then $P=Z \cap C$, since $P \in C$. Suppose that $(4.1)$ is not $\log$ canonical at $P$. By Lemma 4.2, we have $Z \subset \operatorname{Supp}(D)$. By Lemma 3.2 , we may assume that $F \not \subset \operatorname{Supp}(D)$, since $(S,(1-\beta) C+\hat{\alpha}(S,(1-\beta) C) \beta(2 Z+3 F))$ is $\log$ canonical and $2 Z+3 F \sim-K_{S}$. Then $\operatorname{mult}_{P}(D) \leqslant F \cdot D=2$. On the other hand, we have $2 \hat{\alpha}(S,(1-\beta) C) \beta \leqslant$ $\frac{1}{4}+\beta$ and $2 \hat{\alpha}(S,(1-\beta) C) \beta \leqslant 1$. Applying Corollary 3.7 with $n=4$ to (4.1), we get

$8 \hat{\alpha}(S,(1-\beta) C) \beta=\hat{\alpha}(S,(1-\beta) C) \beta(C \cdot D) \geqslant \hat{\alpha}(S,(1-\beta) C) \beta \operatorname{mult}_{P}(C \cdot D)>1+4 \beta$, which contradicts the definition of $\hat{\alpha}(S,(1-\beta) C)$.

Lemma 4.9. Suppose that $4 \leqslant K_{S}^{2} \leqslant 7$, and $P$ is contained in a $(-1)$-curve in $S$. Then (4.1) is log canonical at $P$. 
Proof. See Section 5.

The following result implies Theorem 1.9 modulo Theorem 4.1.

Theorem 4.10. Let $S_{1}$ and $S_{2}$ be smooth del Pezzo surfaces, let $C_{1}$ and $C_{2}$ be smooth curves in $\left|-K_{S_{1}}\right|$ and $\left|-K_{S_{2}}\right|$, respectively. Suppose that there exists a birational morphism $f: S_{2} \rightarrow S_{1}$ such that $f\left(C_{2}\right)=C_{1}$. Then $\hat{\alpha}\left(S_{1},(1-\beta) C_{1}\right) \leqslant \hat{\alpha}\left(S_{2},(1-\beta) C_{2}\right)$ for every $\beta \in(0,1]$ except for the following cases:

(1) $S_{1} \cong \mathbb{P}^{2}, S_{2} \cong \mathbb{F}_{1}$, and $f$ is the blow up of an inflection points of the cubic curve $C_{1} \subset \mathbb{P}^{2}$,

(2) $S_{1} \cong \mathbb{P}^{1} \times \mathbb{P}^{1}, K_{S_{2}}^{2}=7$, and $f$ is the blow up of a point in $C_{1}$.

Proof. Since $f\left(C_{2}\right)=C_{1}$, the morphism $f$ is the blow up of $K_{S_{1}}^{2}-K_{S_{2}}^{2} \geqslant 0$ distinct points on the curve $C_{2}$. Suppose that $\hat{\alpha}\left(S_{1},(1-\beta) C_{1}\right)>\hat{\alpha}\left(S_{2},(1-\right.$ $\beta) C_{2}$ ). Going through all possible cases considered in Section 2, we end up with the following possibilities:

(1) $S_{1} \cong \mathbb{P}^{2}, S_{2} \cong \mathbb{F}_{1}$, and $f$ is the blow up of an inflection points of the cubic curve $C_{1} \subset \mathbb{P}^{2}$,

(2) $S_{1} \cong \mathbb{P}^{1} \times \mathbb{P}^{1}, K_{S_{2}}^{2}=7$, and $f$ is the blow up of a point in $C_{1}$,

(3) $K_{S_{1}}^{2}=4, K_{S_{2}}^{2}=3$, the morphism $f$ is the blow up of a point in $C_{1}$, the curve $C_{1}$ does not contain intersection points of any two lines, for every two conics $Z_{1}$ and $Z_{2}$ in $S_{1}$ such that $Z_{1}+Z_{2} \sim-K_{S_{1}}$, the conics $Z_{1}$ and $Z_{2}$ do not tangent $C_{1}$ at one point, and $S_{2}$ contains an Eckardt point and this point is contained in $C_{2}$,

(4) $K_{S_{1}}^{2}=3, K_{S_{2}}^{2}=2$, the morphism $f$ is the blow up of a point in $C_{1}$, the surface $S_{1}$ contains no Eckardt points, for every line $L$ and every conic $M$ on $S_{1}$ such that $L$ is tangent to $M$ we have $L \cap M \notin C_{1}$, and every irreducible cuspidal curve $T \in\left|-K_{S_{1}}\right|$ intersects $C_{1}$ by at least two point, the linear system $\left|-K_{S_{2}}\right|$ contains a curve with a tacnodal singularity and this tacnodal singular point is contained in $C_{2}$.

The first two cases are indeed possible. Let us show that the last two cases are impossible. Denote by $E$ the $f$-exceptional curve. Then $f(E) \in C_{1}$.

Suppose that $K_{S_{1}}^{2}=4$ and $K_{S_{2}}^{2}=3$. Then $C_{2}$ contains an Eckardt point $O$. Denote by $L_{1}, L_{2}, L_{3}$ the lines in $S_{2}$ that passes through $O$. Then either $E$ is one of these three lines, or $E$ intersects exactly one of them. Without loss of generality, we may assume that either $E=L_{3}$ or $E \cap L_{1}=$ $E \cap L_{3}=\varnothing$. In the former case, $f\left(L_{1}\right)$ and $f\left(L_{2}\right)$ are two conics in $S_{1}$ such that $f\left(L_{1}\right)+f\left(L_{2}\right) \sim-K_{S_{2}}$, and both $f\left(L_{1}\right)$ and $f\left(L_{2}\right)$ tangent the curve $C_{1}=f\left(C_{2}\right)$ at the point $f(P) \in C_{1}$. Since we know that such conics do not exist by assumption, we conclude that $E \cap L_{1}=E \cap L_{3}=\varnothing$. Then $f\left(L_{1}\right)$ and $f\left(L_{2}\right)$ are two lines in $S_{1}$ that both pass through the point $f(P) \in C_{1}$. Such lines do not exist either. Thus, this case is impossible.

Now we suppose that $K_{S_{1}}^{2}=3$ and $K_{S_{2}}^{2}=2$. Let $Z$ be a curve in $\left|-K_{S_{2}}\right|$ such that $Z$ has tacnodal singularity $Q \in C_{2}$. Then $Z=L_{1}+L_{2}$, where $L_{1}$ 
and $L_{2}$ are two $(-1)$-curves in $S_{2}$ that are tangent each other at the point $Q \in C_{2}$. Then either $E$ is one of these two curves, or $E$ intersects exactly one of them. Without loss of generality, we may assume that either $E=L_{2}$ or $E \cap L_{1}=\varnothing$. In the former case, $f\left(L_{1}\right)$ is a cuspidal curve in $\left|-K_{S_{1}}\right|$ whose intersection with the curve $C_{1}$ consists of the point $f(Q)=\operatorname{Sing}\left(f\left(L_{1}\right)\right)$. By assumption, such a cuspidal curve does not exist. Thus, $E \cap L_{1}=\varnothing$. Then $f\left(L_{1}\right)$ is a line, and $f\left(L_{2}\right)$ is a conic. Moreover, the line $f\left(L_{1}\right)$ tangents to $f\left(L_{2}\right)$ at the point $f(Q) \in C_{1}$. The latter is impossible by assumption.

To prove Theorem 4.1, we have to prove that (4.1) is $\log$ canonical at $P$, where $P$ is a point in $C \not \subset \operatorname{Supp}(D)$. The latter follows from Corollary 4.6, Lemmas 4.7, 4.8, 4.9 and

Lemma 4.11. Suppose that $K_{S}^{2} \geqslant 3$, and neither $S \cong \mathbb{P}^{2}$ nor $S \cong \mathbb{P}^{1} \times \mathbb{P}^{1}$. Suppose that $P$ is not contained in any $(-1)$-curve in $S$. If Theorem 4.1 holds for all smooth del Pezzo surfaces of degree $K_{S}^{2}-1$, then (4.1) is log canonical at $P$.

Proof. Suppose that (4.1) is not log canonical at $P$. Let $f: \tilde{S} \rightarrow S$ be a blow up of $P$. Then $\tilde{S}$ is a smooth del Pezzo surface of degree $K_{\tilde{S}}^{2}=K_{S}^{2}-1$, since $P$ is not contained in any $(-1)$-curve in $S$. Denote the $f$-exceptional curve by $E$, denote the proper transform of $C$ on $\tilde{S}$ by $\tilde{C}$, and denote the proper transform of $D$ on $\tilde{S}$ by $\tilde{D}$. Then $\tilde{C} \in\left|-K_{\tilde{S}}\right|$, since $P \in C$. The log pair

$$
\left(\tilde{S},(1-\beta) \tilde{C}+\hat{\alpha}(S,(1-\beta) C) \beta\left(\tilde{D}+\left(\operatorname{mult}_{P}(D)-\frac{1}{\hat{\alpha}(S,(1-\beta) C)}\right) E\right)\right)
$$

is not $\log$ canonical by Lemma 3.3. Let $\tilde{D}^{\prime}=\tilde{D}+\left(\operatorname{mult}_{P}(D)-1\right) E$. Then $\tilde{D}^{\prime} \sim_{\mathbb{R}}-K_{\tilde{S}}$, and $\tilde{D}^{\prime}$ is effective by Lemma 4.2. Furthermore, the log pair $\left(\tilde{S},(1-\beta) \tilde{C}+\hat{\alpha}(S,(1-\beta) C) \beta \tilde{D}^{\prime}\right)$ is not $\log$ canonical, because (4.2) is not $\log$ canonical. This shows that $\hat{\alpha}(S,(1-\beta) C)>\alpha(\tilde{S},(1-\beta) \tilde{C})$. But it follows from Theorem 4.10 that $\hat{\alpha}(\tilde{S},(1-\beta) \tilde{C}) \geqslant \hat{\alpha}(S,(1-\beta) C)$. Thus, we see that $\hat{\alpha}(\tilde{S},(1-\beta) \tilde{C})>\alpha(\tilde{S},(1-\beta) \tilde{C})$. Hence, Theorem 4.1 does not hold for $\tilde{S}$.

This completes the proof of Theorem 4.1 modulo Lemma 4.9.

\section{The Proof of Lemma 4.9}

In this section, we will prove Lemma 4.9. Let us use its notation and assumptions. Then $4 \leqslant K_{S}^{2} \leqslant 7$ and $P$ is a point in $C \not \subset \operatorname{Supp}(D)$ that is contained in a $(-1)$-curve in $S$. Let us denote this $(-1)$-curve by $\mathcal{L}$. We must prove that (4.1) is log canonical at $P$. By Lemma 4.7, we may assume that $\mathcal{L}$ is the only $(-1)$-curve in $S$ that contains $P$. We write $D=a \mathcal{L}+\Omega$, where $a$ is a non-negative real number, and $\Omega$ is an effective $\mathbb{R}$-divisor such that $\mathcal{L} \not \subset \operatorname{Supp}(\Omega)$. By Lemma 4.2, we have $a>0$. Let $x=\operatorname{mult}_{P}(\Omega)$. Then $1+a=\mathcal{L} \cdot \Omega \geqslant x$.

Corollary 5.1. One has $x \leqslant 1+a$. 
Let $\lambda=\hat{\alpha}(S,(1-\beta) C)$. Consider a sequence of 4 blow ups

$$
S_{4} \longrightarrow \pi_{4} \longrightarrow S_{3} \stackrel{\pi_{3}}{\longrightarrow} S_{2} \stackrel{\pi_{2}}{\longrightarrow} S_{1} \stackrel{\pi_{1}}{\longrightarrow} S
$$

such that $\pi_{1}$ is the blow up of the point $P, \pi_{2}$ is the blow up of the intersection point of the $\pi_{1}$-exceptional curve and the proper transform of the curve $C$ on $S_{1}, \pi_{3}$ is the blow up of the intersection point of the $\pi_{2}$-exceptional curve and the proper transform of the curve $C$ on $S_{2}$, and $\pi_{4}$ is the blow up of the intersection point of the $\pi_{3}$-exceptional curve and the proper transform of the curve $C$ on $S_{3}$. Denote by $F_{1}, F_{2}, F_{3}$ and $F_{4}$ the exceptional curves of the blow ups $\pi_{1}, \pi_{2}, \pi_{3}$ and $\pi_{4}$, respectively. Denote by $C^{1}, C^{2}$, $C^{3}$ and $C^{4}$ the proper transforms of the curve $C$ on the surfaces $S_{1}, S_{2}, S_{3}$ and $S_{4}$, respectively. Let $P_{1}=C^{1} \cap F_{1}, P_{2}=C^{2} \cap F_{2}, P_{3}=C^{3} \cap F_{3}$ and $P_{4}=C^{4} \cap F_{4}$. Denote the proper transform of the divisor $\Omega$ on the surfaces $S_{1}, S_{2}, S_{3}$ and $S_{4}$ by $\Omega^{1}, \Omega^{2}, \Omega^{3}$ and $\Omega^{4}$, respectively. Let $x_{1}=\operatorname{mult}_{P_{1}}(\Omega)$, $x_{2}=\operatorname{mult}_{P_{2}}(\Omega)$ and $x_{3}=\operatorname{mult}_{P_{3}}(\Omega)$.

Lemma 5.2. Suppose that (4.1) is not $\log$ canonical at $P$. Then at least one of the following four conditions is not satisfied:

(i) $\lambda \beta(a+x) \leqslant 1$,

(ii) $2 \lambda \beta(a+x)-2 \beta \leqslant 1$ or $\lambda \beta\left(a+x+x_{1}\right)-\beta \leqslant 1$,

(iii) $\lambda \beta\left(a+x+2 x_{1}\right)-3 \beta \leqslant 1$ or $\lambda \beta\left(a+x+x_{1}+x_{2}\right)-2 \beta \leqslant 1$,

(iv) $\lambda \beta\left(a+x+x_{1}+2 x_{2}\right)-4 \beta \leqslant 1$ or $\lambda \beta\left(a+x+x_{1}+x_{2}+x_{3}\right)-3 \beta \leqslant 1$. If $\lambda \beta K_{S}^{2} \leqslant 1+3 \beta$, then at least one of the conditions (i), (ii) or (iii) is not satisfied.

Proof. If conditions (i), (ii), (iii) and (iv) are satisfied, then Corollary 3.8 gives

$$
K_{S}^{2}=D \cdot C \geqslant \operatorname{mult}_{P}(D \cdot C)>\frac{1+4 \beta}{\lambda \beta},
$$

which is impossible, since $\lambda \beta K_{S}^{2} \leqslant 1+4 \beta$ by the definition of $\lambda=\hat{\alpha}(S,(1-$ $\beta) C$ ) for $4 \leqslant K_{S}^{2} \leqslant 7$. Similarly, if conditions (i), (ii), (iii) are satisfied, then $\lambda \beta K_{S}^{2}>1+3 \beta$ by Corollary 3.8 .

Lemma 5.3. Suppose that $K_{S}^{2}=7$. Then (4.1) is log canonical at $P$.

Proof. Suppose that (4.1) is not $\log$ canonical at $P$. Let us use the notation of $\S 2.4$. Without loss of generality, we may assume that either $\mathcal{L}=E_{1}$ or $\mathcal{L}=L$ (but not both).

Suppose that $\mathcal{L}=L$. Since $P \notin E_{1} \cup E_{2}$, the curve $R$ is smooth and irreducible. Since $(S,(1-\beta) C, \lambda \beta(L+2 R))$ is $\log$ canonical and $L+2 R \sim$ $-K_{S}$, we may assume that $R \not \subset \operatorname{Supp}(\Omega)$. Denote the proper transform of the curve $R$ on $S_{1}$ by $R^{1}$, and denote its proper transform on $S_{2}$ by $R^{2}$. Then $3-a-x-x_{1}=R^{2} \cdot \Omega^{2} \geqslant 0$, which gives $a+x+x_{1} \leqslant 3$. Since $x-a \leqslant 1$ by Corollary 5.1, then $x_{1} \leqslant \frac{4}{3}$ and all conditions of Lemma 5.2 are satisfied, giving a contradiction.

We have $\mathcal{L}=E_{1}$. Then $L_{1}$ is irreducible, since $P \notin L$. Since $(S,(1-$ $\left.\beta) C, \lambda \beta\left(2 L_{1}+2 E_{1}+L\right)\right)$ is $\log$ canonical and $2 L_{1}+2 E_{1}+L \sim-K_{S}$, we 
may assume that $L_{1}$ or $L$ is not contained in $\operatorname{Supp}(\Omega)$ by Lemma 3.2. We write $\Omega=b L_{1}+\Delta$, where $b$ is a non-negative real number, and $\Delta$ is an effective $\mathbb{R}$-divisor on $S$ such that $L_{1} \not \subset \operatorname{Supp}(\Delta)$ and $E_{1} \not \subset \operatorname{Supp}(\Delta)$. Then $1-b+a=E_{1} \cdot \Delta \geqslant y$, which gives $b+y \leqslant 1+a$. If $b>0$, then $a \leqslant 1$. Indeed, if $L \not \subset \operatorname{Supp}(\Delta)$, then $1-a=L \cdot \Delta \geqslant 0$.

Denote the proper transform of the divisor $\Delta$ on $S_{1}$ by $\Delta^{1}$, denote the proper transform of the divisor $\Delta$ on $S_{2}$ by $\Delta^{2}$, and denote the proper transform of the divisor $\Delta$ on $S_{3}$ by $\Delta^{3}$. Let $y=\operatorname{mult}_{P}(\Delta), y_{1}=\operatorname{mult}_{P_{1}}\left(\Delta^{1}\right)$, $y_{2}=\operatorname{mult}_{P_{2}}\left(\Delta^{2}\right)$ and $y_{3}=\operatorname{mult}_{P_{3}}\left(\Delta^{3}\right)$. Then $x=b+y$. Since $L_{1} \cdot C=2$, either $\operatorname{mult}_{P}\left(L_{1} \cdot C\right)=1$ or mult $P\left(L_{1} \cdot C\right)=2$. Thus, we have, $x_{2}=y_{2}$ and $x_{3}=y_{3}$.

Suppose that $\operatorname{mult}_{P}\left(L_{1} \cdot C\right)=1$. Then $x_{1}=y_{1}$ and $2-a=L_{1} \cdot \Delta \geqslant y$. We have $b+y \leqslant 1+a$ by Corollary 5.1. If $b>0$, then $a \leqslant 1$. Therefore, we have $\lambda \beta(a+x) \leqslant 1, \lambda \beta\left(a+x+x_{1}\right)-\beta \leqslant 1, \lambda \beta\left(a+x+2 x_{1}\right)-3 \beta \leqslant 1$ and $\lambda \beta\left(a+x+x_{1}+2 x_{2}\right)-4 \beta \leqslant 1$, which contradicts Lemma 5.2.

Thus we see that $\operatorname{mult}_{P}\left(L_{1} \cdot C\right)=2$. Then $x_{1}=y_{1}+b$ and $2-a=$ $L_{1} \cdot \Delta \geqslant y+y_{1}$, which gives $a+y+y_{1} \leqslant 2$. Since $L_{1}$ is tangent to $C$ at the point $P$, we have

$$
\lambda=\hat{\alpha}(S,(1-\beta) C) \leqslant \min \left\{1, \frac{1+2 \beta}{7 \beta}, \frac{1}{3 \beta}\right\} .
$$

Moreover, we have $b+y \leqslant 1+a$ by Corollary 5.1. Furthermore, if $b>0$, then $a \leqslant 1$. This gives $\lambda \beta(a+x) \leqslant 1,2 \lambda \beta(a+x)-2 \beta \leqslant 1, \lambda \beta\left(a+x+x_{1}+x_{2}\right)-2 \beta \leqslant$ 1 and $\lambda \beta\left(a+x+x_{1}+2 x_{2}\right)-4 \beta \leqslant 1$, which is impossible by Lemma 5.2.

Lemma 5.4. Suppose that $K_{S}^{2}=6$. Then (4.1) is log canonical at $P$.

Proof. Suppose that (4.1) is not log canonical at $P$. Let us use the notation of $\S 2.5$. Without loss of generality, we may assume that $\mathcal{L}=E_{1}$. Denote the proper transform of the curve $E_{1}$ on the surface $S_{1}$ by $E_{1}^{1}$. Let $L$ be the proper transform on $S$ of the line in $\mathbb{P}^{2}$ that is tangent to $\pi(C)$ at the point $\pi(P)$. Then $-K_{S} \cdot L=2$, since $P \notin L_{12} \cup L_{13} \cup L_{23}$. Denote the proper transform of the curve $L$ on $S_{1}$ by $L^{1}$, denote the proper transform of the curve $L$ on $S_{2}$ by $L^{2}$, and denote the proper transform of the curve $L$ on $S_{3}$ by $L^{3}$.

We claim that $L \subset \operatorname{Supp}(\Omega)$. Indeed, suppose that $L \not \subset \operatorname{Supp}(\Omega)$. Then $a+x \leqslant 2$, since $2-a=\Omega \cdot L \geqslant x$. But $x \leqslant 1+a$ by Corollary 5.1. Therefore, we have $x_{1} \leqslant x \leqslant \frac{3}{2}$. These inequalities give $\lambda \beta(a+x) \leqslant 1,2 \lambda \beta(a+x)-\beta \leqslant 1$ and $\lambda \beta\left(a+x+2 x_{1}\right)-3 \beta \leqslant 1$. Therefore, $\lambda \beta\left(a+x+x_{1}+2 x_{2}\right)-4 \beta>$ 1 and $6 \lambda \beta>1+3 \beta$ by Lemma 5.2. The former inequality implies that $a+x+x_{1}+2 x_{2}>6$. The latter inequality implies that $L$ is not tangent to $C$ at the point $P$ (see $\S 2.5)$.

Let $Z$ be the proper transform on $S$ of the conic in $\mathbb{P}^{2}$ that passes through the points $\pi\left(E_{1}\right), \pi\left(E_{2}\right), \pi\left(E_{3}\right)$, and is tangent to $\pi(C)$ at the point $\pi(P)$. Then $Z$ is irreducible, $E_{1}+L+Z \sim-K_{S}$ and $-K_{S} \cdot Z=3$, since $L$ is not tangent to $C$ at $P$. Then $\operatorname{mult}_{P}(Z \cdot C) \leqslant 3$, since $-K_{S} \cdot Z=3$. 
We write $\Omega=c Z+\Upsilon$, where $c$ is a non-negative real number, and $\Upsilon$ is an effective $\mathbb{R}$-divisor on $S$ whose support does not contain $Z$. Denote the proper transform of the divisor $\Upsilon$ on $S_{1}$ by $\Upsilon^{1}$, denote the proper transform of the divisor $\Upsilon$ on $S_{2}$ by $\Upsilon^{2}$, and denote the proper transform of the divisor $\Upsilon$ on $S_{3}$ by $\Upsilon^{3}$. Let $z=\operatorname{mult}_{P}(\Upsilon), z_{1}=\operatorname{mult}_{P_{1}}\left(\Upsilon^{1}\right), z_{2}=\operatorname{mult}_{P_{2}}\left(\Upsilon^{2}\right)$, $z_{3}=\operatorname{mult}_{P_{3}}\left(\Upsilon^{3}\right)$. Then $x=c+z, x_{1}=c+z_{1}, x_{3}=z_{3}$. If $\operatorname{mult}_{P}(Z \cdot C)=2$, then $x_{2}=z_{2}$ and $3-a-c-z=Z^{1} \cdot \Upsilon^{1} \geqslant \operatorname{mult}_{P_{1}}\left(Z^{1} \cdot \Upsilon^{1}\right) \geqslant z_{1}$, which implies that

$6<a+x+x_{1}+2 x_{2}=a+z+z_{1}+2 z_{2}+2 c \leqslant 3+2 z_{2}+c \leqslant 3+2 z_{2}+2 c \leqslant 3+2 x \leqslant 6$,

since $z+c \leqslant \frac{3}{2}$ and $a+c+z \leqslant 2$. Thus, we see that $\operatorname{mult}_{P}(Z \cdot C)=3$. Then $x_{2}=c+z_{2}$ and $3-a-c-z-z_{1}=Z^{2} \cdot \Upsilon^{2} \geqslant \operatorname{mult}_{P_{2}}\left(Z^{2} \cdot \Upsilon^{2}\right) \geqslant z_{2}$, which gives $a+c+z+z_{1}+z_{2} \leqslant 3$. Then

$6<a+x+x_{1}+2 x_{2}=a+z+z_{1}+2 z_{2}+3 c<3+z_{2}+2 c \leqslant 3+2 z_{2}+2 c \leqslant 3+2 x \leqslant 6$,

which is absurd. This shows that $L \subset \operatorname{Supp}(\Omega)$.

We write $\Omega=b L+\Delta$, where $b$ is a positive real number, and $\Delta$ is an effective $\mathbb{R}$-divisor on $S$ such that $L \not \subset \operatorname{Supp}(\Delta)$. Let $y=\operatorname{mult}_{P}(\Delta)$. Then $2-a=\Delta \cdot L \geqslant y$. Denote the proper transform of the divisor $\Delta$ on $S_{1}$ by $\Delta^{1}$, denote the proper transform of the divisor $\Delta$ on $S_{2}$ by $\Delta^{2}$, and denote the proper transform of the divisor $\Delta$ on $S_{3}$ by $\Delta^{3}$. Let $y_{1}=\operatorname{mult}_{P_{1}}\left(\Delta^{1}\right)$, $y_{2}=\operatorname{mult}_{P_{2}}\left(\Delta^{2}\right)$ and $y_{3}=\operatorname{mult}_{P_{3}}\left(\Delta^{3}\right)$. Then $x=b+y, x_{2}=y_{2}$ and $x_{3}=y_{3}$, which implies that $b+y \leqslant 1+a$ by Corollary 5.1. Then

$$
\left(S_{1},(1-\beta) C^{1}+\lambda \beta a E_{1}^{1}+\lambda \beta b L^{1}+\lambda \beta \Delta^{1}+(\lambda \beta(a+b+y)-\beta) F_{1}\right)
$$

is not $\log$ canonical at some point $Q_{1} \in F_{1}$ by Lemma 3.3 .

We claim that $L$ is tangent to $C$ at the point $P$. Indeed, suppose that $L$ is not tangent to $C$ at $P$. Then $x_{1}=y_{1}$. Let $Z$ be the proper transform on $S$ of the conic in $\mathbb{P}^{2}$ that passes through $\pi\left(E_{1}\right), \pi\left(E_{2}\right), \pi\left(E_{3}\right)$, and is tangent to $\pi(C)$ at $\pi(P)$. Then $Z$ is irreducible and $-K_{S} \cdot Z=3$. Moreover, we have $E_{1}+L+Z \sim-K_{S}$, and the $\log$ pair $\left(S,(1-\beta) C+\lambda \beta\left(E_{1}+L+Z\right)\right)$ is $\log$ canonical. Thus, we may assume that $Z \not \subset \operatorname{Supp}(D)$ by Lemmas 3.2. Then $3-a-b-y=Z^{1} \cdot \Delta^{1} \geqslant \operatorname{mult}_{P_{1}}\left(Z^{1} \cdot \Delta^{1}\right) \geqslant y_{1}$. Since we also have $b+y \leqslant 1+a, a+y \leqslant 2, x=y+b, x_{1}=y_{1}$ and $x_{2}=y_{2}$, we see that

$$
\begin{aligned}
& \lambda \beta y_{1} \leqslant 1, \quad \lambda \beta(a+b+y)-\beta \leqslant \lambda \beta\left(a+b+y+y_{1}\right)-\beta \leqslant 1, \\
& \lambda \beta\left(a+b+y+2 y_{1}\right)-3 \beta \leqslant 1, \quad \lambda \beta\left(a+b+y_{1}+2 y_{2}\right)-4 \beta \leqslant 1 .
\end{aligned}
$$

In particular, (5.1) is $\log$ canonical at every point of $F_{1}$ that is different from $Q_{1}$ by Lemma 3.3. If $Q_{1} \neq L^{1} \cap F_{1}$ and $Q_{1} \neq P_{1}$, then $\lambda \beta(a+y)=$ $F_{1} \cdot\left(\lambda \beta\left(a E_{1}+\Delta^{1}\right)\right)>1$, by Theorem 3.4. But $\lambda \beta(a+y) \leqslant 1$, since $a+y \leqslant 2$. This shows that $Q_{1}=L^{1} \cap F_{1}$ or $Q_{1}=P_{1}$. Since $b-a+y \leqslant 1$ and $a+b+y+y_{1} \leqslant 3$, we have $b+y \leqslant 2$. So, if $Q_{1}=L^{1} \cap F_{1}$, then

$$
1<\lambda \beta F_{1} \cdot\left(b L^{1}+\Delta^{1}\right)=\lambda \beta(b+y) \leqslant 2 \lambda \beta \leqslant 1,
$$


by Theorem 3.4. If $Q_{1}=P_{1}$, then $6=D \cdot C>\frac{1+4 \beta}{\lambda \beta}$ by (5.2) and Theorem 3.6. The latter contradicts $6 \lambda \beta \leqslant 1+4 \beta$.

We see that $L$ is tangent to $C$ at the point $P$. Then $x_{1}=y_{1}+b$ and

$$
\lambda \leqslant \min \left\{1, \frac{1+2 \beta}{5 \beta}, \frac{1}{2 \beta}\right\},
$$

which gives $6 \lambda \beta \leqslant 1+3 \beta$. Moreover, we have $a+y+y_{1} \leqslant 2$, because $2-a-y-y_{1}=L^{2} \cdot \Delta^{2} \geqslant 0$. Furthermore, since $2 L+L_{23}+E_{1} \sim-K_{S}$ and $\left(S,(1-\beta) C+\lambda \beta\left(2 L+L_{23}+E_{1}\right)\right.$ is $\log$ canonical, we may assume that $L_{23} \not \subset$ $\operatorname{Supp}(\Delta)$ by Lemma 3.2. This gives us $b \leqslant 1$, because $1-b=\Delta \cdot L_{23} \geqslant 0$. Since $L+L_{12}+L_{13}+2 E_{1} \sim-K_{S}$ and $\left(S,(1-\beta) C+\lambda \beta\left(L+L_{12}+L_{13}+2 E_{1}\right)\right)$ is $\log$ canonical, we may assume that $L_{12} \not \subset \operatorname{Supp}(\Delta)$ or $L_{13} \not \subset \operatorname{Supp}(\Delta)$ by Lemma 3.2. If $L_{12} \not \subset \operatorname{Supp}(\Delta)$, then $1-a=\Delta \cdot L_{12} \geqslant 0$, which gives $a \leqslant 1$. Similarly, we get $a \leqslant 1$ if $L_{13} \not \subset \operatorname{Supp}(\Delta)$. Thus, we have

$$
b \leqslant 1, \quad b-a+y \leqslant 1,
$$$$
a+y+y_{1} \leqslant 2,
$$

which implies that $\lambda \beta(a+b+y)-\beta \leqslant 1$. In particular, (5.1) is log canonical at every point of $F_{1}$ that is different from $Q_{1}$ by Lemma 3.3. If $Q_{1} \neq P_{1}$ and $Q_{1} \neq E_{1}^{1} \cap F_{1}$, then $\lambda \beta y=\lambda \beta \Delta^{1} \cdot F_{1}>1$ by Theorem 3.4. The latter is impossible, since $\lambda \beta y \leqslant 2 \lambda \beta \leqslant 1$ by (5.3). If $Q_{1}=E_{1}^{1} \cap F_{1}$, then

$$
1<E_{1}^{1} \cdot\left(\lambda \beta \Delta^{1}+(\lambda \beta(a+b+y)-\beta) F_{1}\right)=\lambda \beta(1+2 a)-\beta
$$

by Theorem 3.4. The latter is impossible, since $\lambda \beta(1+2 a)-\beta \leqslant 3 \lambda \beta-\beta \leqslant 1$ by (5.3). Thus, we see that $Q_{1}=P_{1}$.

By (5.3), one has $a+2 b+y+y_{1} \leqslant 4$. This implies that $\lambda \beta(a+2 b+y+$ $\left.y_{1}\right)-2 \beta \leqslant 1$. Then

$$
\left(S_{2},(1-\beta) C^{2}+\lambda \beta b L^{2}+\lambda \beta \Delta^{2}+(\lambda \beta(a+b+y)-\beta) F_{1}^{2}+\left(\lambda \beta\left(a+2 b+y+y_{1}\right)-2 \beta\right) F_{2}\right)
$$

is not $\log$ canonical at a unique point $Q_{2} \in F_{2}$ by Lemma 3.3. If $Q_{2} \notin$ $L^{2} \cup F_{1}^{2} \cup C^{2}$, then $\lambda \beta y_{2}=\lambda \beta \Delta^{2} \cdot F_{2}>1$ by Theorem 3.4, which is impossible, since $\lambda \beta y_{2} \leqslant 1$ by (5.3). Similarly, if $Q_{2}=F_{2} \cap L^{2}$, then $\lambda \beta\left(b+y_{2}\right)=$ $\lambda \beta\left(b L^{2}+\Delta^{2}\right) \cdot F_{2}>1$ by Theorem 3.4, which is impossible, because $b+y_{2} \leqslant$ $b+y \leqslant 2$ by (5.3). If $Q_{2}=F_{2} \cap F_{1}^{2}$, then

$$
\lambda \beta\left(y+y_{1}+a+b\right)-\beta=\left(\lambda \beta \Delta^{2}+(\lambda \beta(a+b+y)-\beta) F_{1}^{2}\right) \cdot F_{2}>1
$$

by Theorem 3.4, which is impossible, since $y+y_{1}+a+b \leqslant 3$ by (5.3). Then $Q_{2}=P_{2}$.

We have $\lambda \beta\left(a+2 b+y+y_{1}+y_{2}\right)-3 \beta \leqslant 1$, since $a+2 b+y+y_{1}+y_{2} \leqslant 5$ by (5.3). Then

$$
\left(S_{3},(1-\beta) C^{3}+\lambda \beta \Delta^{3}+\left(\lambda \beta\left(a+2 b+y+y_{1}\right)-2 \beta\right) F_{2}^{3}+\left(\lambda \beta\left(a+2 b+y+y_{1}+y_{2}\right)-3 \beta\right) F_{3}\right) .
$$

is not $\log$ canonical at a unique point $Q_{3} \in F_{3}$ by Lemma 3.3. If $Q_{3} \notin$ $F_{2}^{3} \cup C^{3}$, then $\lambda \beta y_{3}=\lambda \beta \Delta^{3} \cdot F_{3}>1$ by Theorem 3.4 , which is impossible, 
because $\lambda \beta y_{3} \leqslant 1$ by (5.3). If $Q_{3}=F_{3} \cap F_{2}^{3}$, then Theorem 3.4 gives

$1<F_{2}^{3} \cdot\left(\lambda \beta \Delta^{3}+\left(\lambda \beta\left(a+2 b+y+y_{1}+y_{2}\right)-3 \beta\right) F_{3}\right)=\lambda \beta\left(a+2 b+y+2 y_{1}\right)-3 \beta \leqslant 5 \lambda \beta-3 \beta$,

which is impossible, since $a+2 b+y+2 y_{1} \leqslant 5$ by (5.3). Thus, we see that $Q_{3}=$ $P_{3}$. By Theorem 3.6 (iv), we have $6=D \cdot C \geqslant \operatorname{mult}_{P}(D \cdot C)>\frac{1+3 \beta}{\lambda \beta}$. The latter is impossible, since we already proved earlier that $6 \lambda \beta \leqslant 1+3 \beta$.

Lemma 5.5. Suppose that $K_{S}^{2}=5$. Then (4.1) is log canonical at $P$.

Proof. Suppose that (4.1) is not log canonical at $P$. Let us use the notation of $\oint 2.5$. Then $\lambda=\min \left\{1, \frac{1}{2 \beta}\right\}$. This implies that $5 \lambda \beta \leqslant 1+3 \beta$. By Lemma 5.2, at least one of the conditions (i), (ii) and (iii) in Lemma 5.2 is not satisfied. In particular, if $a+x \leqslant 2$, then $\lambda \beta\left(a+x+2 x_{1}\right)-3 \beta>1$.

Without loss of generality, we may assume that $\mathcal{L}=L_{12}$. Let $B_{3}$ be the proper transform on $S$ of the line in $\mathbb{P}^{2}$ that passes through $\pi(P)$ and $\pi\left(E_{3}\right)$, and let $B_{4}$ be the proper transform on $S$ of the line in $\mathbb{P}^{2}$ that passes through $\pi(P)$ and $\pi\left(E_{4}\right)$. Since $L_{12}+B_{3}+B_{4} \sim-K_{S}$ and $(S,(1-\beta) C+$ $\left.\lambda \beta\left(L_{12}+B_{3}+B_{4}\right)\right)$ is log canonical, we may assume that at least one curve among $B_{3}$ and $B_{4}$ is not contained in $\operatorname{Supp}(\Omega)$. Intersecting this curve with $\Omega$, we get $a+x \leqslant 2$. Then $\lambda \beta\left(a+x+2 x_{1}\right)-3 \beta>1$. This implies that $a+x+2 x_{1}>5$.

Denote the proper transform of the curve $B_{3}$ on the surface $S_{1}$ by $B_{3}^{1}$, and denote the proper transform of the curve $B_{4}$ on the surface $S_{1}$ by $B_{4}^{1}$. Recall $P_{1}=C^{1} \cap F_{1}$.

Suppose that $P_{1} \notin B_{3}^{1} \cup B_{4}^{1}$. Then $B_{3}$ and $B_{4}$ do not tangent $C$ at $P$. Let $R$ be the proper transform on $S$ of the line in $\mathbb{P}^{2}$ that is tangent to $\pi(C)$ at the point $\pi(P)$, let $R_{1}$ be the proper transform on $S$ of the conic in $\mathbb{P}^{2}$ that tangents to $\pi(C)$ at the point $\pi(P)$ and passes through the points $\pi\left(E_{2}\right), \pi\left(E_{3}\right)$ and $\pi\left(E_{4}\right)$, and let $R_{2}$ be the proper transform on $S$ of the conic in $\mathbb{P}^{2}$ that tangents to $\pi(C)$ at the point $\pi(P)$ and passes through the points $\pi\left(E_{1}\right), \pi\left(E_{3}\right)$ and $\pi\left(E_{4}\right)$. Since $P_{1} \notin B_{3}^{1} \cup B_{4}^{1}$, the curves $R_{1}$ and $R_{2}$ are irreducible. Hence $\frac{1}{2} L_{12}+\frac{1}{2} R+\frac{1}{2} R_{1}+\frac{1}{2} R_{2} \sim_{\mathbb{R}}-K_{S}$ and $\left(S,(1-\beta) C+\lambda \beta\left(\frac{1}{2} L_{12}+\frac{1}{2} R+\frac{1}{2} R_{1}+\frac{1}{2} R_{2}\right)\right)$ is $\log$ canonical. By Lemma 3.2, we may assume that one curve among $R, R_{1}$ and $R_{2}$ is not contained in $\operatorname{Supp}(D)$. Denote this curve by $Z$, and denote its proper transform on $S_{1}$ by $Z^{1}$. Then $P_{1} \in Z^{1}$ and $3-a-x=Z^{1} \cdot \Omega^{1} \geqslant x_{1}$, which is impossible, since $a+x \leqslant 2$ and $a+x+2 x_{1}>5$.

We see that $P_{1}=B_{3}^{1} \cap F_{1}$ or $P_{1}=B_{4}^{1} \cap F_{1}$. Without loss of generality, we may assume that $P_{1}=B_{3}^{1} \cap F_{1}$. Then $B_{3} \subset \operatorname{Supp}(\Omega)$, since otherwise we would have $2-a-x=B_{3}^{1} \cdot \Omega^{1} \geqslant x_{1}$, which is impossible, since $a+x \leqslant 2$. We write $\Omega=b B_{3}+\Delta$, where $b \in \mathbb{R}_{>0}$ and $\Delta$ is an effective $\mathbb{R}$-divisor on $S$ such that $B_{3} \not \subset \operatorname{Supp}(\Delta)$. Denote the proper transform of the divisor $\Delta$ on $S_{1}$ by $\Delta^{1}$. Let $y=\operatorname{mult}_{P}(\Delta)$ and $y_{1}=$ mult $_{P_{1}}\left(\Delta^{1}\right)$. Then $x=b+y$ and $x_{1}=b+y_{1}$. We have $b-a+y \leqslant 1$ by Corollary 5.1 and $a+b+y=a+x \leqslant 2$, which implies a contradiction $a+x+2 x_{1} \leqslant 2+2 y+2 b \leqslant 5$. 
Lemma 5.6. Suppose that $K_{S}^{2}=4$. Then (4.1) is log canonical at $P$.

Proof. Suppose that (4.1) is not $\log$ canonical at $P$. Let us use the notation $\S 2.7$. Then $\lambda \beta<\frac{2}{3}$. Without loss of generality, we may assume that $P \in E$. Then $P=E \cap C$. By Lemma 4.7, the point $P$ is not contained in any other $(-1)$-curve. By Lemma 4.2, we have $E \subset \operatorname{Supp}(D)$.

The log pair $\left(S,(1-\beta) C+\lambda \beta\left(\frac{3}{2} E+\frac{1}{2}\left(E_{1}+E_{2}+E_{3}+E_{4}+E_{5}\right)\right)\right)$ is $\log$ canonical and $\frac{3}{2} E+\frac{1}{2}\left(E_{1}+E_{2}+E_{3}+E_{4}+E_{5}\right) \sim_{\mathbb{R}}-K_{S}$. By Lemma 3.2, we may assume that $\operatorname{Supp}(\Omega)$ does not contain one curve among $E_{1}, E_{2}, E_{3}$, $E_{4}, E_{5}$. Intersecting this curve with $\Omega$, we get $a \leqslant 1$. Let $L_{1}, L_{2}, L_{3}, L_{4}, L_{5}$ be the proper transforms on $S$ of the lines in $\mathbb{P}^{2}$ that pass through $\pi(P)$ and $\pi\left(E_{1}\right), \pi\left(E_{2}\right), \pi\left(E_{3}\right), \pi\left(E_{4}\right), \pi\left(E_{5}\right)$, respectively. Then $\frac{2}{3} E+\frac{1}{3}\left(L_{1}+L_{2}+L_{3}+\right.$ $\left.L_{4}+L_{5}\right) \sim_{\mathbb{R}}-K_{S}$, and $\left(S,(1-\beta) C+\lambda \beta\left(\frac{2}{3} E+\frac{1}{3}\left(L_{1}+L_{2}+L_{3}+L_{4}+L_{5}\right)\right)\right)$ is $\log$ canonical. By Lemma 3.2, we may assume that $\operatorname{Supp}(\Omega)$ does not contain one curve among $L_{1}, L_{2}, L_{3}, L_{4}, L_{5}$. Intersecting this curve with $\Omega$, we get $a+x \leqslant 2$. Recall that $a \leqslant 1$ by Corollary 5.1 . Thus, we have

$$
a \leqslant 1, \quad x-a \leqslant 1, \quad a+x \leqslant 2,
$$

which implies that $x \leqslant \frac{3}{2}$ and $\lambda \beta(a+x)-\beta \leqslant 1$. In particular, we have $\lambda \beta x \leqslant 1$.

Denote the proper transform of the curve $E$ on $S_{1}$ by $E^{1}$. Then $\lambda \beta(a+$ $x)-\beta \leqslant 1$, since $a+x \leqslant 2$. Thus, the $\log$ pair $\left(S_{1},(1-\beta) C^{1}+\lambda \beta a E^{1}+\right.$ $\left.\lambda \beta \Omega^{1}+(\lambda \beta(a+x)-\beta) F_{1}\right)$ is not log canonical at the unique point $Q_{1} \in F_{1}$ by Lemma 3.3. Note that $\lambda \beta(a+x)-\beta>0$ by Lemma 3.1. Moreover, either $Q_{1}=P_{1}$ or $Q_{1}=E^{1} \cap F_{1}$, since otherwise we would have $\lambda x=\lambda \beta \Omega^{1} \cdot F_{1}>1$ by Theorem 3.4. If $Q_{1}=E^{1} \cap F_{1}$, then Theorem 3.9 implies

$$
\lambda \beta(1+a-x)=\lambda \beta \Omega^{1} \cdot E^{1}>2(1+\beta-\lambda \beta(x+a))
$$

or $\lambda \beta x=\lambda \beta \Omega^{1} \cdot F_{1}>2(1-\lambda \beta a)$. The former inequality gives $\lambda \beta(1+3 a+x)>$ $2+2 \beta$, which is impossible since $1+3 a+x \leqslant 5$ by (5.4). The latter inequality gives that $\lambda \beta(x+2 a)>2$, which is impossible since $x+2 a \leqslant 3$ by (5.4). Thus, we see that $Q_{1}=P_{1}$.

Let $R$ be the proper transform on $S$ of a line in $\mathbb{P}^{2}$ that is tangent to $\pi(C)$ at the point $\pi(P)$. Then either $-K_{S} \cdot R=3$ or $-K_{S} \cdot R=2$. Moreover, $-K_{S} \cdot R=3$ if and only if $\pi(R)$ does not contain any of the points $\pi\left(E_{1}\right)$, $\pi\left(E_{2}\right), \pi\left(E_{3}\right), \pi\left(E_{4}\right), \pi\left(E_{5}\right)$.

Suppose that $-K_{S} \cdot R=2$. Without loss of generality, we may assume that $R=L_{1}$. We write $\Omega=b L_{1}+\Delta$, where $b$ is a non-negative real number, and $\Delta$ is an effective $\mathbb{R}$-divisor on $S$ whose support does not contain the curve $L_{1}$. Denote the proper transform of the curve $L_{1}$ on $S_{1}$ by $L_{1}^{1}$, and denote the proper transform of $\Delta$ on $S_{1}$ by $\Delta^{1}$. Let $y=\operatorname{mult}_{P}(\Delta)$ and $y_{1}=\operatorname{mult}_{P_{1}}\left(\Delta^{1}\right)$. Then $x=y+b$. Since $\left(S,(1-\beta) C+\lambda \beta\left(E+E_{1}+L_{1}\right)\right)$ is $\log$ canonical and $E+E_{1}+L_{1} \sim-K_{S}$, we may assume that $b=0$ or $\operatorname{Supp}(\Delta)$ does not contain $E_{1}$ by Lemma 3.2. Thus, if $b \neq 0$, then $1-a-b=\Delta \cdot E_{1} \geqslant 0$. With (5.4), this gives $y+2 b \leqslant 2$ and $2+a+y+2 b \leqslant \frac{9}{2}$. On the other hand, 
we have $2-a-y=\Delta^{1} \cdot L_{1}^{1} \geqslant y_{1}$, which implies that $a+2 y_{1} \leqslant 2$, since $y \geqslant y_{1}$. Thus, we see that $y_{1} \leqslant 1$. Then mult $_{P_{1}}\left((1-\beta) C^{1}+\lambda \beta \Delta^{1}\right)=1-\beta+\lambda \beta y_{1} \leqslant 1$. Applying Theorem 3.9, we see that

$1-\beta+\lambda \beta(2-a-y)=\left((1-\beta) C^{1}+\lambda \beta \Delta^{1}\right) \cdot L_{1}^{1}>2(1+\beta-\lambda \beta(a+b+y))$ or $1-\beta+\lambda \beta y=\left((1-\beta) C^{1}+\lambda \beta \Delta^{1}\right) \cdot F_{1}>2(1-\lambda \beta b)$. This gives $\lambda \beta(2+a+$ $y+2 b)>1+3 \beta$ or $\lambda \beta(y+2 b)>1+\beta$. The former inequality is impossible, because $2+a+y+2 b \leqslant \frac{9}{2}$. The latter inequality is also impossible, because $y+2 b \leqslant 2$.

We have $-K_{S} \cdot R=3$. Then $R$ is irreducible and $R+E \sim-K_{S}$. Since $(S,(1-\beta) C+\lambda \beta(R+E))$ is $\log$ canonical, we may assume that $R \not \subset \operatorname{Supp}(\Omega)$ by Lemma 3.2. Denote the proper transform of the curve $R$ on the surface $S_{1}$ by $R^{1}$. Then $3-2 a-x=\Omega^{1} \cdot R^{1} \geqslant x_{1}$, which gives $x+x_{1}+2 a \leqslant 3$. Then $\lambda \beta\left(a+x+x_{1}\right)-2 \beta \leqslant 1$ by (5.4). Thus, the log pair

$$
\left(S_{2},(1-\beta) C^{2}+\lambda \beta \Omega^{2}+(\lambda \beta(a+x)-\beta) F_{1}^{2}+\left(\lambda \beta\left(a+x+x_{1}\right)-2 \beta\right) F_{2}\right)
$$

is not $\log$ canonical at a unique point $Q_{2} \in F_{2}$ by Lemma 3.3. Note that $\lambda \beta\left(a+x+x_{1}\right)-2 \beta>0$ by Lemma 3.1. If $Q_{2} \neq P_{2}$ and $Q_{2} \neq F_{1}^{2} \cap F_{2}$, then Theorem 3.4 gives $\lambda \beta x_{1}=\lambda \beta \Omega^{2} \cdot F_{2}>1$, which is impossible, since $\lambda \beta x_{1} \leqslant \lambda \beta x \leqslant 1$ by (5.4). If $Q_{2}=F_{1}^{2} \cap F_{2}$, then Theorem 3.4 gives

$$
\lambda \beta(a+2 x)-2 \beta \geqslant\left(\lambda \beta \Omega^{2}+\left(\lambda \beta\left(a+x+x_{1}\right)-2 \beta\right) F_{2}\right) \cdot F_{1}^{2}>1
$$

which is impossible, since $a+2 x \leqslant \frac{7}{2}$, by (5.4). Hence, we see that $Q_{2}=P_{2}$.

One has $\lambda \beta\left(a+x+x_{1}+x_{2}\right)-3 \beta \leqslant 1$ by (5.4), since $x+x_{1}+2 a \leqslant 3$ and $x_{2} \leqslant x_{1} \leqslant x$. Thus, it follows from Lemma 3.3 that

$$
\left(S_{3},(1-\beta) C^{3}+\lambda \beta \Omega^{3}+\left(\lambda \beta\left(a+x+x_{1}\right)-2 \beta\right) F_{2}^{3}+\left(\lambda \beta\left(a+x+x_{1}+x_{2}\right)-3 \beta\right) F_{3}\right)
$$

is not $\log$ canonical at a unique point $Q_{3} \in F_{3}$. Note that $\lambda \beta\left(a+x+x_{1}+\right.$ $\left.x_{2}\right)-3 \beta>0$ by Lemma 3.1. If $Q_{3} \neq P_{3}$ and $Q_{3} \neq F_{2}^{3} \cap F_{3}$, then Theorem 3.4 gives $\lambda \beta x_{2}=\lambda \beta \Omega^{3} \cdot F_{3}>1$, which is impossible, since $\lambda \beta x_{2} \leqslant \lambda \beta x \leqslant 1$ by (5.4). If $Q_{3}=F_{2}^{3} \cap F_{3}$, then Theorem 3.4 gives

$$
\lambda \beta\left(a+x+2 x_{1}\right)-3 \beta=\left(\lambda \beta \Omega^{3}+\left(\lambda \beta\left(a+x+x_{1}+x_{2}\right)-3 \beta\right) F_{3}\right) \cdot F_{2}^{3}>1
$$

which contradicts $(5.4)$, since $x+x_{1}+2 a \leqslant 3$. Thus, we have $Q_{3}=P_{3}$. Then Theorem 3.4 gives

$$
\beta \geqslant 4 \lambda \beta-3 \beta=C^{3} \cdot\left(\lambda \beta \Omega^{3}+\left(\lambda \beta\left(a+x+x_{1}+x_{2}\right)-3 \beta\right) F_{3}\right)>1,
$$

which is impossible, since $\beta \in(0,1]$.

This completes the proof of Lemma 4.9 .

Acknowledgements. The work was supported by the grant of Russian Science Foundation (project No. 15-11-20036). We thank Yanir Rubinstein and Gabor Székelyhidi for useful discussions. 


\section{REFERENCES}

[1] I. Cheltsov, Log canonical thresholds of del Pezzo surfaces, Geom. Funct. Anal. 18 (2008), 1118-1144.

[2] I. Cheltsov, Del Pezzo surfaces and local inequalities, to appear in Proceedings of the Trento conference "Groups of Automorphisms in Birational and Affine Geometry", (2012), Springer.

[3] I. Cheltsov, J. Park, J. Won, Affine cones over smooth cubic surfaces, preprint, arXiv:1303.2648 (2013).

[4] I. Cheltsov, C. Shramov, Log canonical thresholds of smooth Fano threefolds, with an appendix by J.-P. Demailly, Russian Math. Surv. 63 (2008), 859-958.

[5] I. Cheltsov, Y. Rubinstein, Asymptotically log Fano varieties, preprint, arXiv:1308.2503 (2013).

[6] X-X. Chen, S. Donaldson, S. Sun, Kahler-Einstein metrics and stability, preprint, arXiv:1210.7494 (2012).

[7] D. Coman, V. Guedj, Quasiplurisubharmonic Green functions, Journal de Math. Pures et Appliquées 92 (2009), 456-475.

[8] A. Corti, J. Kollár, K. Smith Rational and nearly rational varieties, Cambridge University Press, (2003).

[9] S. Donaldson, Kähler metrics with cone singularities along a divisor, in: Essays on Mathematics and its applications (P. Pardalos et al., Eds.), Springer (2012), 49-79.

[10] T. Jeffres, R. Mazzeo, Y. Rubinstein, Kähler-Einstein metrics with edge singularities, with an appendix by C. Li and Y. Rubinstein, to appear in Ann. of Math., preprint, arxiv:1105.5216 (2011).

[11] J. Kollár Rational Curves on Algebraic Varieties, Springer, (1996).

[12] C. Li, S. Sun, Conical Kahler-Einstein metrics revisited, preprint, arxiv:1207.5011 (2012).

[13] J. Martinez-Garcia, Dynamic Alpha-Invariants of del Pezzo Surfaces with Boundary, Ph.D. Thesis, University of Edinburgh, (2013).

[14] J. Martinez-Garcia, Log canonical thresholds of Del Pezzo Surfaces in characteristic p, Manuscripta Mathematica 145 (2014), 89-110

[15] Y. Odaka, S. Sun, Testing log K-stability by blowing up formalism, preprint, arxiv:1112.1353 (2011).

[16] Y. Shi, On the $\alpha$-invariants of cubic surfaces with Eckardt points, Adv. Math. 225 (2010), 1285-1307.

[17] V. Shokurov, Smoothness of a general anticanonical divisor on a Fano variety, Izv. Akad. Nauk SSSR Ser. Mat. 43 (1979), 430-441.

[18] J. Song, The $\alpha$-invariant on toric Fano manifolds, Amer. J. Math. 127 (2005), 12471259.

[19] G. Tian, On Kähler-Einstein metrics on certain Kähler manifolds with $c_{1}(M)>0$, Invent. Math. 89 (1987), 225-246.

[20] G. Tian, On Calabi's conjecture for complex surfaces with positive first Chern class, Invent. Math. 101 (1990), 101-172.

[21] G. Tian, K-stability and Kähler-Einstein metrics, preprint, arxiv:1211.4669 (2012).

[22] G. Székelyhidi, A remark on conical Kähler-Einstein metrics, Math. Res. Lett. 20 (2013), 581-590. 